\title{
A METHOD FOR FINDING A MINIMAL POINT OF THE LATTICE IN CUBIC NUMBER FIELDS
}

\author{
By
}

Kan KaneKo

\begin{abstract}
We give a method for finding a minimal point adjacent to 1 of the reduced lattice in cubic number fields using an isotropic vector of the quadratic form and two-dimensional lattice.
\end{abstract}

\section{Introduction}

Let $K$ be a cubic algebraic number field of negative discriminant. It is known that to find all the minimal points of a reduced lattice $\mathscr{R}$ of $K$, it is sufficient to know how to find a minimal point adjacent to 1 in any reduced lattice of $K$ (refer to Definition 1.1 for a rigorous definition). Williams, Cormack and Seah [6] utilized the two-dimensional lattice obtained from a reduced lattice $\mathscr{R}$ to find a minimal point adjacent to 1 in $\mathscr{R}$ (the definition of such a two-dimensional lattice is forthcoming in Section 2). Moreover, Adam [1] utilized an isotropic vector of the quadratic form obtained from a basis of reduced lattice $\mathscr{R}$ (the definition of such a quadratic form is forthcoming in Section 4). Later, Lahlou and Farhane [5] generalise the Adam's method.

In this paper, we shall prove six theorems which give candidates of a minimal point adjacent to 1 in a reduced lattice $\mathscr{R}$. In each case of the theorems, the maximum number of candidates $\varphi \in \mathscr{R}$ such that we must check whether $F(\varphi)<1$ or not is at most four. Also, such six theorems contain all the occuring cases.

Definition 1.1. (1) Let $1, \beta, \gamma \in K$ be independent over $\mathbf{Q}$. We say that $\mathscr{R}=\langle 1, \beta, \gamma\rangle=\mathbf{Z}+\mathbf{Z} . \beta+\mathbf{Z} . \gamma$ is a lattice of $K$ with basis $\{1, \beta, \gamma\}$.

AMS 2010 Mathematics Subject Classification: 11R16, 11R27.

Key words and phrases: cubic fields, Voronoi algorithm, fundamental units.

Received August 5, 2013.

Revised November 26, 2013. 
(2) For $\alpha \in \mathscr{R}$ we define $F(\alpha)=\frac{N_{K}(\alpha)}{\alpha}=\alpha^{\prime} \alpha^{\prime \prime}$, where $N_{K}$ denotes the norm of $K$ over $\mathbf{Q}$, and $\alpha^{\prime}$ and $\alpha^{\prime \prime}$ the conjugates of $\alpha$.

(3) Let $\mathscr{R}$ be a lattice of $K$, and let $\varphi(>0) \in \mathscr{R}$. We say that $\varphi$ is a minimal point of $\mathscr{R}$ if for all $\alpha$ in $\mathscr{R}$ such that $0<\alpha<\varphi$ we have $F(\alpha)>F(\varphi)$.

(4) Let $\mathscr{R}$ be a lattice of $K$ and $\varphi, \psi \in \mathscr{R}$ be a minimal point. We say that $\psi$ is a minimal point adjacent to $\varphi$ in $\mathscr{R}$ if $\psi=\min \{\alpha \in \mathscr{R} ; \varphi<\alpha$, $F(\varphi)>F(\alpha)\}$.

(5) If $\mathscr{R}$ is a lattice of $K$ in which 1 is a minimal point, we call $\mathscr{R}$ a reduced lattice.

\section{Basis of Reduced Lattice (I)}

Definition 2.1. Let $\alpha \in K$. We define $Y_{\alpha}:=\operatorname{Re} \alpha^{\prime}, Z_{\alpha}:=\operatorname{Im} \alpha^{\prime}, X_{\alpha}:=$ $\alpha-Y_{\alpha}$. Let $\lambda \in K, \quad \mu \in K \backslash \mathbf{Q}$. We define $\omega_{1}(\lambda, \mu):=-\left(Z_{\lambda} / Z_{\mu}\right), \quad \omega_{2}(\lambda, \mu):=$ $-Y_{\lambda}-\omega_{1}(\lambda, \mu) Y_{\mu}$.

REMARK. In [6] $Y_{\alpha}=\operatorname{Im} \alpha^{\prime}, Z_{\alpha}=\operatorname{Re} \alpha^{\prime}$.

Proposition 2.2. Let $\alpha \in K, c \in \mathbf{Z}$. Then

(1) $F(\alpha)=Y_{\alpha}^{2}+Z_{\alpha}^{2}$.

(2) $\alpha \notin \mathbf{Q} \Rightarrow Y_{\alpha}, X_{\alpha} \in K-\mathbf{Q}, Z_{\alpha} \notin \mathbf{Q}$.

(3) $K \ni 1, \lambda, \mu$ are independent over $\mathbf{Q} \Rightarrow \omega_{1}(\lambda, \mu) \notin \mathbf{Q}$.

(4) $K \ni 1, \lambda, \mu$ are independent over $\mathbf{Q} \Rightarrow 1, X_{\lambda}, X_{\mu}$ are independent over $\mathbf{Q}$.

(5) $K \ni 1, \lambda, \mu$ are independent over $\mathbf{Q} \Rightarrow \operatorname{det}\left(\begin{array}{cc}X_{\lambda} & X_{\mu} \\ Z_{\lambda} & Z_{\mu}\end{array}\right) \neq 0$.

(6) Let $\alpha \notin \mathbf{Q}$. Then

(i) $-1<Y_{\alpha+c}<1 \Leftrightarrow c=\left[-Y_{\alpha}\right]$ or $\left[-Y_{\alpha}\right]+1$,

(ii) $Y_{\left[-Y_{\alpha}\right]+\alpha}<0, \quad Y_{\left[-Y_{\alpha}\right]+1+\alpha}>0$,

(iii) $\left|Y_{\left[-Y_{\alpha}\right]+\alpha}\right|<1 / 2$ or $\left|Y_{\left[-Y_{\alpha}\right]+1+\alpha}\right|<1 / 2$.

Proof. (3) Let $K=\mathbf{Q}(\theta)$ and $\lambda=a_{0}+a_{1} \theta+a_{2} \theta^{2} \quad\left(a_{i} \in \mathbf{Q}\right), \mu=b_{0}+b_{1} \theta+$ $b_{2} \theta^{2}\left(b_{i} \in \mathbf{Q}\right)$. Then we have

$$
\begin{aligned}
Z_{\lambda} & =\frac{1}{2 i}\left(\lambda^{\prime}-\lambda^{\prime \prime}\right)=\frac{1}{2 i}\left\{a_{1}\left(\theta^{\prime}-\theta^{\prime \prime}\right)+a_{2}\left(\theta^{\prime 2}-\theta^{\prime \prime 2}\right)\right\} \\
& =\frac{1}{2 i}\left(\theta^{\prime}-\theta^{\prime \prime}\right)\left\{a_{1}+a_{2}\left(\theta^{\prime}+\theta^{\prime \prime}\right)\right\}=Z_{\theta}\left\{a_{1}+\left(T_{K / \mathbf{Q}} \theta\right) a_{2}-a_{2} \theta\right\} \quad\left(i^{2}=-1\right)
\end{aligned}
$$


Similarly we have $Z_{\mu}=Z_{\theta}\left\{b_{1}+\left(T_{K / \mathbf{Q}} \theta\right) b_{2}-b_{2} \theta\right\}$. Suppose that

$$
\omega_{1}(\lambda, \mu)=-\frac{Z_{\lambda}}{Z_{\mu}}=-\frac{a_{1}+p a_{2}-a_{2} \theta}{b_{1}+p b_{2}-b_{2} \theta}=r \in \mathbf{Q} \quad\left(p=T_{K / \mathbf{Q}} \theta\right) .
$$

Then we have

$r\left(b_{1}+p b_{2}-b_{2} \theta\right)=-\left(a_{1}+p a_{2}-a_{2} \theta\right), \quad r b_{1}+r p b_{2}+a_{1}+p a_{2}-\left(r b_{2}+a_{2}\right) \theta=0$.

Hence $r b_{2}+a_{2}=0, r b_{1}+a_{1}=0$, so $a_{0}+r b_{0}-\lambda-r \mu=0$.

Since $1, \lambda, \mu$ are independent over $\mathbf{Q}$, we have reached a contradiction.

Therefore we have $\omega_{1}(\lambda, \mu) \notin \mathbf{Q}$.

(5) Since $1, \lambda, \mu$ are independent over $\mathbf{Q}$, by algebraic number theory $\operatorname{det}\left(\begin{array}{ccc}1 & \lambda & \mu \\ 1 & \lambda^{\prime} & \mu^{\prime} \\ 1 & \lambda^{\prime \prime} & \mu^{\prime \prime}\end{array}\right) \neq 0$. Moreover, $\operatorname{det}\left(\begin{array}{ccc}1 & \lambda & \mu \\ 1 & \lambda^{\prime} & \mu^{\prime} \\ 1 & \lambda^{\prime \prime} & \mu^{\prime \prime}\end{array}\right)=2 i\left(X_{\lambda} Z_{\mu}-X_{\mu} Z_{\lambda}\right)$.

Therefore we have $X_{\lambda} Z_{\mu}-X_{\mu} Z_{\lambda} \neq 0$.

Otheres are easily deduced from definitions.

Definition 2.3. Let $\mathscr{R}$ be a reduced lattice of $K$. For $\mathscr{R} \ni \alpha$ we define

$$
\alpha_{(1)}:=\left[-Y_{\alpha}\right]+\alpha, \quad \alpha_{(2)}:=\left[-Y_{\alpha}\right]+1+\alpha, \quad \alpha_{(3)}:=\left\{\begin{array}{ll}
\alpha_{(1)} & \text { if }\left|Y_{\alpha_{(1)}}\right|<1 / 2 \\
\alpha_{(2)} & \text { if }\left|Y_{\alpha_{(2)}}\right|<1 / 2
\end{array},\right.
$$

$\alpha_{(0)}:=\alpha-[\alpha]$, where $[\ldots]$ is the greatest integer function.

Note that $\left|Z_{\alpha}\right|<\sqrt{3} / 2 \Rightarrow F\left(\alpha_{(3)}\right)<1$.

Let $\mathscr{R}=\langle 1, \beta, \gamma\rangle$ be a reduced lattice of $K$. Let $\tau: K \rightarrow \mathbf{R}^{2}$ be the Q-linear map defined by $\alpha^{\tau}=\left(X_{\alpha}, Z_{\alpha}\right)$. Note that for $\alpha_{1}, \alpha_{2} \in \mathscr{R}, \alpha_{1}^{\tau}=\alpha_{2}^{\tau} \Leftrightarrow$ there exists some $c \in \mathbf{Z}$ such that $\alpha_{2}=c+\alpha_{1}$. Let $L:=\mathscr{R}^{\tau}=\left\langle\beta^{\tau}, \gamma^{\tau}\right\rangle$. By Proposition 2.2,(5) $L$ is a two-dimensional lattice. Moreover, by Proposition 2.2,(3)(4) $L$ has the following property $(\Delta)$ :

$$
\text { ( } \Delta) \quad L \cap(\{0\} \times \mathbf{R})=L \cap(\mathbf{R} \times\{0\})=\{(0,0)\} .
$$

Now we prepare two lemmas about the two-dimensional lattice which has property $(\Delta)$ from Delone's supplement I in [2].

Definition 2.4. Let $L\left(\subset \mathbf{R}^{2}\right)$ be a two-dimensional lattice which has property $(\Delta)$. (1) For $\mathbf{R}^{2} \ni S=\left(S_{u}, S_{v}\right) \neq(0,0)$ we define $C(S):=\left\{(u, v) \in \mathbf{R}^{2}\right.$; $\left.|u|<\left|S_{u}\right|,|v|<\left|S_{v}\right|\right\}$. Then we say that $S \in L$ is a minimal point of $L$ if 
$L \cap C(S)=\{(0,0)\}$. The system of all the minimal points of $L$ we denote by $M(L)$. We put $M(L)_{>0}:=\left\{P \in M(L) ; P_{u}>0\right\}$.

(2) Let $S\left(S_{u}>0\right), Q\left(Q_{u}>0\right) \in L$ be a minimal point of $L$. We say that $Q$ is a minimal point adjacent to $S$ in $L$ if $Q_{u}=\min \left\{P_{u} ; P \in L, S_{u}<P_{u},\left|S_{v}\right|>\left|P_{v}\right|\right\}$.

Lemma 2.5. Let $L\left(\subset \mathbf{R}^{2}\right)$ be a two-dimensional lattice which has property $(\Delta)$. Let $L \ni S, Q\left(S_{u}>0, Q_{u}>0\right)$. Then $Q$ is a minimal point adjacent to $S$ in $L$ if and only if $L=\langle S, Q\rangle, S_{u}<Q_{u},\left|S_{v}\right|>\left|Q_{v}\right|, S_{v} Q_{v}<0$.

Proof. From Theorem XI,XII,XIII in [2, p. 467-469]. (cf. Theorem 4.1 in [9]).

Lemma 2.6. Let $L\left(\subset \mathbf{R}^{2}\right)$ be a two-dimensional lattice which has property $(\Delta)$ and let $E, G, H \in L$. We assume that $G$ is a minimal point adjacent to $E$ and that $H$ is a minimal point adjacent to $G$. Then we have $H=E+\left[-E_{v} / G_{v}\right] G$.

Proof. From supplement I, Section 3, 34 in [2, p. 470].

Proposition 2.7. Let $\mathscr{R}$ be a reduced lattice of $K$, and let $L:=\mathscr{R}^{\tau}$. Then there exists a basis $\{1, \lambda, \mu\}$ of $\mathscr{R}$ such that $\lambda^{\tau}$ is a minimal point adjacent to $\mu^{\tau}$ in $L, 0<X_{\lambda}, F\left(\lambda_{(3)}\right)<1, F\left(\mu_{(3)}\right)>1$.

Proof. Let $\mathscr{R}=\langle 1, \beta, \gamma\rangle$. For $\varepsilon>0$, we shall consider a rectangular neighbourhood of $(0,0)$, i.e. $W(\varepsilon, \sqrt{3} / 2)=\left\{(u, v) \in \mathbf{R}^{2} ;|u|<\varepsilon,|v|<\sqrt{3} / 2\right\}$. By Minkowski's convex body theorem, there exists $\varepsilon>0$ such that $L \cap W(\varepsilon, \sqrt{3} / 2) \neq$ $\{(0,0)\}$. We take such a $\varepsilon>0$ and fix it. We put $W=W(\varepsilon, \sqrt{3} / 2)$. Then there exists $Q=\left(Q_{u}, Q_{v}\right) \in L \cap W$ such that $Q_{u}=\min \left\{P_{u} ; P \in L \cap W, 0<P_{u}\right\}$. Note that such a $Q \in L$ is uniquely-determined. We have $L \cap C(Q)=\{(0,0)\}$. Hence $Q$ is a minimal point of $L$. There exists $S \in L$ such that $Q$ is a minimal point adjacent to $S$ in $L$. By Lemma 2.5, $\{S, Q\}$ is a basis of $L$. Since both $\{S, Q\}$ and $\left\{\beta^{\tau}, \gamma^{\tau}\right\}$ are a basis of $L$, there exists $\left(\begin{array}{ll}p & q \\ r & s\end{array}\right) \in G L_{2}(\mathbf{Z})$ such that $(Q S)=\left(\begin{array}{ll}\beta^{\tau} & \gamma^{\tau}\end{array}\right)\left(\begin{array}{ll}p & q \\ r & s\end{array}\right)$. We have $Q=p \beta^{\tau}+r \gamma^{\tau}=(p \beta+r \gamma)^{\tau}$. Similarly, we have $S=(q \beta+s \gamma)^{\tau}$. We define $\lambda, \mu \in K$ by $(\lambda \mu)=\left(\begin{array}{ll}\beta & \gamma\end{array}\right)\left(\begin{array}{ll}p & q \\ r & s\end{array}\right)$. Then we have $\mathscr{R}=\langle 1, \lambda, \mu\rangle, Q=\lambda^{\tau}, S=\mu^{\tau}$. Since $Q=\left(Q_{u}, Q_{v}\right)=\lambda^{\tau}=\left(X_{\lambda}, Z_{\lambda}\right)$, from $\left|Z_{\lambda}\right|<$ $\sqrt{3} / 2$, we have $F\left(\lambda_{(3)}\right)<1$. From this, if we put $\mathscr{R}_{F}:=\left\{\alpha \in \mathscr{R} ; \alpha^{\tau} \in M(L)_{>0}\right.$, $\left.F\left(\alpha_{(3)}\right)<1\right\}$, then $\mathscr{R}_{F} \neq \varnothing$. Let $W(\varepsilon, 1):=\left\{(u, v) \in \mathbf{R}^{2} ;|u|<\varepsilon,|v|<1\right\}$. As 
$W(\varepsilon, \sqrt{3} / 2) \subset W(\varepsilon, 1)$, we have $1<\left|\mathscr{R}_{F}^{\tau} \cap W(\varepsilon, 1)\right|<\infty$. Hence there exists $\lambda^{\tau} \in \mathscr{R}_{F}^{\tau} \cap W(\varepsilon, 1)$ such that $X_{\lambda}=\min \left\{X_{\alpha} ; \alpha^{\tau} \in \mathscr{R}_{F}^{\tau} \cap W(\varepsilon, 1)\right\}$. Since $F\left(\alpha_{(3)}\right)<1 \Rightarrow$ $\left|Z_{\alpha}\right|<1$, it is easily seen that $X_{\lambda}=\min \left\{X_{\alpha} ; \alpha^{\tau} \in \mathscr{R}_{F}^{\tau} \cap W(\varepsilon, 1)\right\}=\min \left\{X_{\alpha}\right.$; $\left.\alpha^{\tau} \in \mathscr{R}_{F}^{\tau}\right\}=\min \left\{X_{\alpha} ; \alpha \in \mathscr{R}_{F}\right\}$. For this $\lambda$, there exists $\mu \in \mathscr{R}$ such that $\lambda^{\tau}$ is a minimal point adjacent to $\mu^{\tau}$ in $L$. Moreover, for such a $\mu$ we have $F\left(\mu_{(3)}\right)>1$.

REMARK. Such a basis in Proposition 2.7 is easily found by modified version of Algorithm (A) in [6, p. 581].

Definition 2.8. Let $\mathscr{R}$ be a reduced lattice of $K$, and let $L:=\mathscr{R}^{\tau}$. We say that $\lambda \in \mathscr{R}$ is a $F$-point of $M(L)_{>0}$ if $\lambda \in \mathscr{R}_{F}, X_{\lambda}=\min \left\{X_{\alpha} ; \alpha \in \mathscr{R}_{F}\right\}$.

Lemma 2.9. Let $\mathscr{R}$ be a reduced lattice of $K$. If $0<X_{\lambda}, F\left(\lambda_{(3)}\right)<1$, then we have $0<\lambda_{(1)}$.

ProOF. We assume that $0<X_{\lambda}, \quad F\left(\lambda_{(3)}\right)<1$. From $0<X_{\lambda}=X_{\lambda_{(2)}}=$ $\lambda_{(2)}-Y_{\lambda_{(2)}}$, we have $\lambda_{(2)}>Y_{\lambda_{(2)}}>0$. Hence we have $\lambda_{(2)}>0$. Suppose that $\lambda_{(1)}<0$. We have $0<\lambda_{(2)}=\lambda_{(1)}+1<1$, so $-1<\lambda_{(1)}<0$. Since $\mathscr{R}$ is a reduced lattice of $K$, we have $F\left(\lambda_{(2)}\right)>1$. Hence we have $\lambda_{(3)}=\lambda_{(1)}$, so $F\left(\lambda_{(1)}\right)<1$. From this, $F\left(-\lambda_{(1)}\right)<1$. Since $\mathscr{R}$ is a reduced lattice of $K$, we have reached a contradiction. Therefore, we have $\lambda_{(1)}>0$.

THEOREM 2.10. Let $\mathscr{R}$ be a reduced lattice of $K$. Then there exists a basis $\{1, \lambda, \mu\}$ of $\mathscr{R}$ such that

(a) $0<\lambda<1,-1 / 2<\mu, F(\mu)>1,2\left|Y_{\mu}\right|<1,0<X_{\mu}<X_{\lambda}, 0<\omega_{1}(\lambda, \mu)<1$,

(b) $\omega_{2}(\lambda, \mu)>0$,

(c) $F\left(\left[\omega_{2}\right]+\lambda\right)<1$ or $F\left(\left[\omega_{2}\right]+1+\lambda\right)<1$.

Proof. By Proposition 2.7, we can take a basis $\{1, \lambda, \mu\}$ of $\mathscr{R}$ such that $\lambda^{\tau}$ is a minimal point adjacent to $\mu^{\tau}$ in $L, 0<X_{\lambda}, F\left(\lambda_{(3)}\right)<1, F\left(\mu_{(3)}\right)>1, \lambda$ is a $F$-point of $M(L)_{>0}$. Clearly, $\mathscr{R}=\left\langle 1, \lambda_{(0)}, \mu_{(3)}\right\rangle$.

(a) Clearly we have $0<\lambda_{(0)}<1, F\left(\mu_{(3)}\right)>1,2\left|Y_{\mu_{(3)}}\right|<1,0<X_{\mu_{(3)}}=X_{\mu}<$ $X_{\lambda_{(0)}}=X_{\lambda}$. From $0<X_{\mu}=X_{\mu_{(3)}}=\mu_{(3)}-Y_{\mu_{(3)}}$, we have $-1 / 2<\mu_{(3)}$. From Remark 2.11 bellow, we have $0<\omega_{1}(\lambda, \mu)<1$. Since $\omega_{1}\left(\lambda_{(0)}, \mu_{(3)}\right)=-\left(Z_{\lambda_{(0)}} / Z_{\mu_{(3)}}\right)=$ $-\left(Z_{\lambda} / Z_{\mu}\right)=\omega_{1}(\lambda, \mu)$, we have $0<\omega_{1}\left(\lambda_{(0)}, \mu_{(3)}\right)<1$.

(b) Proof of " $\omega_{2}\left(\lambda_{(0)}, \mu_{(3)}\right)>0$ ". 
(i) The case $\lambda_{(1)}=\left[-Y_{\lambda}\right]+\lambda>1 . \quad \lambda_{(1)}=\left[-Y_{\lambda}\right]+\lambda=\left[-Y_{\lambda_{(0)}}\right]+\lambda_{(0)}>1$. Hence $-Y_{\lambda_{(0)}}>1$. From this and from $0<\omega_{1}<1, \quad\left|Y_{\mu_{(3)}}\right|<1 / 2$ we have $\omega_{2}\left(\lambda_{(0)}, \mu_{(3)}\right)=-Y_{\lambda_{(0)}}-\omega_{1}\left(\lambda_{(0)}, \mu_{(3)}\right) Y_{\mu_{(3)}}>0$.

(ii) The case $\lambda_{(1)}=\left[-Y_{\lambda}\right]+\lambda<1$. By Lemma 2.9, we have $\lambda_{(1)}>0$. From $0<\lambda_{(1)}<1$, we have $F\left(\lambda_{(1)}\right)>1$ because $\mathscr{R}$ is a reduced lattice of $K$. Therefore we have $F\left(\lambda_{(2)}\right)<1$. Since $F\left(\lambda_{(1)}\right)>1$, we have $Y_{\lambda_{(1)}}<-1 / 2$. Note that $\lambda_{(1)}=\lambda_{(0)}$. Hence from $Y_{\lambda_{(0)}}=Y_{\lambda_{(1)}}<-1 / 2$ and from $0<\omega_{1}<1,\left|Y_{\mu_{(3)}}\right|<1 / 2$ we have $\omega_{2}\left(\lambda_{(0)}, \mu_{(3)}\right)=-Y_{\lambda_{(0)}}-\omega_{1}\left(\lambda_{(0)}, \mu_{(3)}\right) Y_{\mu_{(3)}}>0$.

(c) Proof of " $F\left(\left[\omega_{2}\right]+\lambda_{(0)}\right)<1$ or $F\left(\left[\omega_{2}\right]+1+\lambda_{(0)}\right)<1$ ".

(i) The case $Y_{\mu_{(3)}}<0$. Since $\omega_{2}-\left(-Y_{\lambda_{(0)}}\right)=-\omega_{1} Y_{\mu_{(3)}}>0$, we have $-Y_{\lambda_{(0)}}<\omega_{2}$. From this and $\left|-\omega_{1} Y_{\mu_{(3)}}\right|<1 / 2$, we have $\left[\omega_{2}\right]=\left[-Y_{\lambda_{(0)}}\right]$ or $\left[-Y_{\lambda_{(0)}}\right]+1$. Note that $\left[\omega_{2}\right]=\left[-Y_{\lambda_{(0)}}\right]+1 \Rightarrow 0<\left[-Y_{\lambda_{(0)}}\right]+1-\left(-Y_{\lambda_{(0)}}\right)<1 / 2 \Rightarrow$ $0<Y_{\lambda_{(2)}}=\left[-Y_{\lambda_{(0)}}\right]+1+Y_{\lambda_{(0)}}<1 / 2$. Hence if $\left[\omega_{2}\right]=\left[-Y_{\lambda_{(0)}}\right]+1$, then we have $\lambda_{(3)}=\lambda_{(2)}$. Therefore, we have " $\left[\omega_{2}\right]+\lambda_{(0)}=\left[-Y_{\lambda_{(0)}}\right]+\lambda_{(0)}=\lambda_{(1)},\left[\omega_{2}\right]+1+\lambda_{(0)}$ $=\lambda_{(2)}$ " or " $\left[\omega_{2}\right]+\lambda_{(0)}=\left[-Y_{\lambda_{(0)}}\right]+1+\lambda_{(0)}=\lambda_{(2)}, F\left(\lambda_{(2)}\right)<1$ ".

(ii) The case $Y_{\mu_{(3)}}>0$. Since $\omega_{2}-\left(-Y_{\lambda_{(0)}}\right)=-\omega_{1} Y_{\mu_{(3)}}<0$, we have $-Y_{\lambda_{(0)}}>\omega_{2}$. From this and $\left|-\omega_{1} Y_{\mu_{(3)}}\right|<1 / 2$, we have $\left[\omega_{2}\right]=\left[-Y_{\lambda_{(0)}}\right]$ or $\left[-Y_{\lambda_{(0)}}\right]-1$. Note that $\left[\omega_{2}\right]=\left[-Y_{\lambda_{(0)}}\right]-1 \Rightarrow 0<-Y_{\lambda_{(0)}}-\left[-Y_{\lambda_{(0)}}\right]<1 / 2 \Rightarrow-1 / 2$ $<Y_{\lambda_{(1)}}=\left[-Y_{\lambda_{(0)}}\right]+Y_{\lambda_{(0)}}<0$. Hence if $\left[\omega_{2}\right]=\left[-Y_{\lambda_{(0)}}\right]-1$, then we have $\lambda_{(3)}=\lambda_{(1)}$. Therefore we have " $\left[\omega_{2}\right]+\lambda_{(0)}=\left[-Y_{\lambda_{(0)}}\right]+\lambda_{(0)}=\lambda_{(1)},\left[\omega_{2}\right]+1+\lambda_{(0)}$ $=\lambda_{(2)}$ " or " $\left[\omega_{2}\right]+1+\lambda_{(0)}=\lambda_{(1)}, F\left(\lambda_{(1)}\right)<1$ ".

REMARK 2.11. Let $\mathscr{R}=\langle 1, \beta, \gamma\rangle, 0<X_{\gamma}<X_{\beta}$. Then $\gamma^{\tau}$ is a minimal point adjacent to $\beta^{\tau}$ in $L \Leftrightarrow 0<\omega_{1}(\beta, \gamma)<1$.

\section{Basis of Reduced Lattice (II)}

Definition 3.1. Let $\mathscr{R}$ be a lattice of $K$, and let $\{1, N, M\}$ be a basis of $\mathscr{R}$. We say that $\{1, N, M\}$ is normalized provided that

$$
0<X_{M}<X_{N}, \quad\left|Z_{M}\right|>1 / 2, \quad\left|Z_{N}\right|<1 / 2, \quad Z_{M} \cdot Z_{N}<0 .
$$

We quote Williams [9], Theorem 8.1 as Theorem 3.2 for our convenience.

THEOREM 3.2 (Williams [9], Theorem 8.1). Let $\mathscr{R}$ be a reduced lattice with the normalized basis $\{1, N, M\}$. If $\theta_{g}=x+y N+z M(x, y, z \in \mathbf{Z})$ is the minimal point adjacent to 1 , then $(y, z) \in\{(1,0),(0,1),(1,1),(1,-1),(2,1)\}$. 
In this paper, $\theta_{g}$ denotes the minimal point adjacent to 1 of any reduced lattice $\mathscr{R}$. We shall consider the relationship between $F$-point and the normalized basis.

THEOREM 3.3. Let $\mathscr{R}$ be a reduced lattice with the normalized basis $\{1, N, M\}$. If $\mathscr{R}=\langle 1, \lambda, \mu\rangle, \lambda^{\tau}$ is adjacent to $\mu^{\tau}, \lambda$ is a F-point of $M(L)_{>0}$ $\left(L=\mathscr{R}^{\tau}\right)$, then $\lambda^{\tau}$ must be one of $N^{\tau},(N-M)^{\tau}, M^{\tau}$. Moreover,

(1) The case $\lambda^{\tau}=(N-M)^{\tau}: N^{\tau}=(d+1) \lambda^{\tau}+\mu^{\tau}, M^{\tau}=d \lambda^{\tau}+\mu^{\tau}$,

(2) The case $\lambda^{\tau}=M^{\tau}: N^{\tau}=d \lambda^{\tau}+\mu^{\tau}$,

where $d=d(\lambda, \mu)=\left[1 / \omega_{1}(\lambda, \mu)\right]$.

Proof. Recall that $\mathscr{R}_{F}=\left\{\alpha \in \mathscr{R} ; \alpha^{\tau} \in M(L)_{>0}, F\left(\alpha_{(3)}\right)<1\right\}, \quad X_{\lambda}=\min \left\{X_{\alpha} ;\right.$ $\left.\alpha \in \mathscr{R}_{F}\right\}$. By Lemma 2.5 and Definition 3.1, we have $N \in \mathscr{R}_{F}$. Hence, we have $X_{\lambda} \leq X_{N}$. Since $L=\left\langle N^{\tau}, M^{\tau}\right\rangle=\left\langle\lambda^{\tau}, \mu^{\tau}\right\rangle$, there exists $a, b \in \mathbf{Z}$ such that $\lambda^{\tau}=$ $a N^{\tau}+b M^{\tau}$

(i) The case $a<0$. Since $X_{\lambda}>0$, we have $b>0$. Moreover, since $\left|Z_{\lambda}\right|=$ $\left|a Z_{N}+b Z_{M}\right|=|a| \cdot\left|Z_{N}\right|+b \cdot\left|Z_{M}\right|<1$ and $1 / 2<\left|Z_{M}\right|$, we have $b \leq 1$. Therefore $b=1$. Hence $X_{\lambda}=a X_{N}+b X_{M}=a X_{N}+X_{M}=X_{M}-|a| \cdot X_{N}<0$. Therefore the case (i) is impossible.

(ii) The case $a=0$. Since $X_{\lambda}=a X_{N}+b X_{M}=b X_{M}$, we have $b>0$. Since $\left|Z_{\lambda}\right|=b\left|Z_{M}\right|$, we have $b=1$. [i.e. $(a, b)=(0,1)$ ]

(iii) The case $a \geq 1, b \leq 0$. Since $\left|Z_{\lambda}\right|=a\left|Z_{N}\right|+|b| \cdot\left|Z_{M}\right|<1$, we have $|b| \leq 1$.

1) The case $b=-1$. Since $X_{\lambda}=a X_{N}-X_{M}=(a-1) X_{N}+\left(X_{N}-X_{M}\right)$, if $a \geq 2$, then we have $X_{\lambda}>X_{N}$, which is impossible. Therefore, we have $a=1$. [i.e. $(a, b)=(1,-1)]$

2) The case $b=0$. Since $X_{\lambda}=a X_{N}=(a-1) X_{N}+X_{N}$, if $a \geq 2$, then we have $X_{\lambda}>X_{N}$, which is impossible. Therefore, we have $a=1$. [i.e. $(a, b)=$ $(1,0)]$

(iv) The case $a \geq 1, b \geq 1$. We have $X_{\lambda}=a X_{N}+b X_{M}>X_{N}$, which is impossible. Therefore, the case (iv) is impossible.

By (i) to (iv), we conclude that $\lambda^{\tau}=a N^{\tau}+b M^{\tau}=M^{\tau}$ or $(N-M)^{\tau}$ or $N^{\tau}$.

(a) The case $\left|Z_{\lambda}\right|<1 / 2$. Since $\left|Z_{\mu}\right|>\sqrt{3} / 2>1 / 2$, we have $\lambda^{\tau}=N^{\tau}$, $\mu^{\tau}=M^{\tau}$.

(b) The case $\left|Z_{\lambda}\right|>1 / 2$. Since $\lambda^{\tau} \neq N^{\tau}$, we have $0<X_{\lambda}<X_{N}$. Hence we have $\lambda^{\tau}=(N-M)^{\tau}$ or $M^{\tau}$.

(b-1) The case $\lambda^{\tau}=(N-M)^{\tau}$. We have

(1.1) $X_{\lambda}=X_{N-M}<X_{M}<X_{N}$. 
Because if $X_{M}<X_{\lambda}=X_{N-M}<X_{N}$, then from $X_{M}<X_{N-M},\left|Z_{M}\right|<\left|Z_{N-M}\right|$, we have $L \cap C\left((N-M)^{\tau}\right)=L \cap\left\{(u, v) \in \mathbf{R}^{2} ;|u|<X_{N-M},|v|<\left|Z_{N-M}\right|\right\} \ni M^{\tau} \neq$ $(0,0)$. Since $\lambda^{\tau}=(N-M)^{\tau} \in L$ is a minimal point, we have reached a contradiction. Therefore we have $X_{\lambda}=X_{N-M}<X_{M}<X_{N}$. By Remark 2.11 we have $0<\omega_{1}(N, M)<1$. Since $\omega_{1}(M, N-M)=\frac{1}{\omega_{1}(N, M)+1}$, we have $0<$ $\omega_{1}(M, N-M)<1$. From this, if $X_{N-M}<X_{M}$, then $M^{\tau}$ is adjacent to $(N-M)^{\tau}$. Note that $\mathscr{R}=\langle 1, M, N-M\rangle$. Hence we have

(1.2) $X_{N-M}<X_{M} \Leftrightarrow M^{\tau}$ is adjacent to $(N-M)^{\tau}$.

Since $M^{\tau}$ is a minimal point adjacent to $\lambda^{\tau}$, and $\lambda^{\tau}$ is a minimal point adjacent to $\mu^{\tau}$, by Lemma 2.6 we have $M^{\tau}=\mu^{\tau}+\left[-\left(Z_{\mu} / Z_{\lambda}\right)\right] \lambda^{\tau}$. We put $d=\left[-\left(Z_{\mu} / Z_{\lambda}\right)\right]=\left[1 / \omega_{1}(\lambda, \mu)\right]$. We have $M^{\tau}=\mu^{\tau}+d \lambda^{\tau}$. From $\lambda^{\tau}=N^{\tau}-M^{\tau}$, we have $N^{\tau}=\mu^{\tau}+(d+1) \lambda^{\tau}$. Therefore we obtain formulas: $M^{\tau}=d \lambda^{\tau}+\mu^{\tau}$, $N^{\tau}=(d+1) \lambda^{\tau}+\mu^{\tau}$.

(b-2) The case $\lambda^{\tau}=M^{\tau}$.

Since $N^{\tau}$ is a minimal point adjacent to $\lambda^{\tau}$, and $\lambda^{\tau}$ is a minimal point adjacent to $\mu^{\tau}$, by Lemma 2.6 we have $N^{\tau}=\mu^{\tau}+\left[-\left(Z_{\mu} / Z_{\lambda}\right)\right] \lambda^{\tau}=\mu^{\tau}+d \lambda^{\tau}$. Therefore we obtain formulas: $M^{\tau}=\lambda^{\tau}, N^{\tau}=d \lambda^{\tau}+\mu^{\tau}$.

COROLlary 3.4. Let $\mathscr{R}$ be a reduced lattice with basis $\{1, \lambda, \mu\}$ such that $\lambda^{\tau}$ is adjacent to $\mu^{\tau}, \lambda$ is a F-point of $M(L)_{>0}\left(L=\mathscr{R}^{\tau}\right)$. If $\theta_{g}=x+y \lambda+z \mu$ $(x, y, z \in \mathbf{Z})$, then

the case $\lambda^{\tau}=N^{\tau}:(y, z) \in\{(1,0),(1,1),(1,-1),(2,1)\}$,

the case $\lambda^{\tau}=(N-M)^{\tau}:(y, z) \in\{(1,0),(d, 1),(d+1,1),(2 d+1,2),(3 d+2,3)\}$,

the case $\lambda^{\tau}=M^{\tau}:(y, z) \in\{(1,0),(d, 1),(d+1,1),(2 d+1,2),(d-1,1)\}$, where $d=\left[1 / \omega_{1}(\lambda, \mu)\right] \geq 1$.

Proof. From Theorem 3.2.

REMARK 3.5. Since $1 /(d+1)<\omega_{1}<1 / d$, we have

$$
\begin{aligned}
& {\left[d \omega_{1}\right]=\left[(d-1) \omega_{1}\right]=0, \quad\left[(d+1) \omega_{1}\right]=1,} \\
& 1 \leq\left[(2 d+1) \omega_{1}\right] \leq 2, \quad 2 \leq\left[(3 d+2) \omega_{1}\right] \leq 4 .
\end{aligned}
$$

THEOREM 3.6. Let $\mathscr{R}$ be a reduced lattice with basis $\{1, \lambda, \mu\}$ such that $F(\mu)>1,2\left|Y_{\mu}\right|<1,0<X_{\mu}<X_{\lambda}, 0<\omega_{1}(\lambda, \mu)<1, F\left(\lambda_{(3)}\right)<1$.

Then $\lambda^{\tau}$ must be one of $N^{\tau},(N-M)^{\tau}, M^{\tau}$. Moreover, if $\lambda^{\tau}=(N-M)^{\tau}$ or $M^{\tau}$, then $\lambda$ is a F-point of $M(L)_{>0}\left(L=\mathscr{R}^{\tau}\right)$. 
Proof. At first, we note that $\lambda^{\tau}$ is adjacent to $\mu^{\tau}$. Also $\lambda \in \mathscr{R}_{F}$. From $2\left|Y_{\mu}\right|<1, \mu=\mu_{(3)}$.

(a) The case $\left|Z_{\lambda}\right|<1 / 2$. Since $F\left(\mu_{(3)}\right)=F(\mu)>1$, we have $\left|Z_{\mu}\right|>\sqrt{3} / 2>$ $1 / 2$. Hence we have $\lambda^{\tau}=N^{\tau}, \mu^{\tau}=M^{\tau}$.

(b) The case $\left|Z_{\lambda}\right|>1 / 2$. Let $\lambda^{*}$ be a $F$-point of $M(L)_{>0}$. So we have $X_{\lambda^{*}} \leq X_{\lambda}$. We shall show that $\lambda^{* \tau}=\lambda^{\tau}$. Suppose that $\lambda^{* \tau} \neq \lambda^{\tau}$.

(i) The case $\lambda^{\tau} \neq M^{\tau}$. We have

(i-1) $X_{\lambda^{*}}<X_{\mu}<X_{\lambda}<X_{M}<X_{N}$.

Since $\left|Z_{\lambda^{*}}\right|>1 / 2$, by Theorem 3.3, we have $\lambda^{* \tau}=M^{\tau}$ or $(N-M)^{\tau}$. Hence $\lambda^{* \tau}=(N-M)^{\tau}$. By (1.1) in the proof of Theorem 3.3, we have $X_{\lambda^{*}}=X_{N-M}<$ $X_{M}$. From (i-1), we have $X_{\lambda^{*}}=X_{N-M}<X_{\mu}<X_{\lambda}<X_{M}<X_{N}$. Since $M^{\tau}$ is adjacent to $(N-M)^{\tau}$, we have reached a contradiction.

(ii) The case $\lambda^{\tau}=M^{\tau}$. Since $\lambda^{* \tau} \neq \lambda^{\tau}$, by Theorem 3.3, we have $\lambda^{* \tau}=$ $(N-M)^{\tau}$. By (1.1) in the proof of Theorem 3.3, we have $X_{\lambda^{*}}=X_{N-M}<X_{M}$. Hence we have $X_{\lambda^{*}}=X_{N-M}<X_{\mu}<X_{\lambda}=X_{M}<X_{N}$. Since $M^{\tau}$ is adjacent to $(N-M)^{\tau}$, we have reached a contradiction.

By (i)(ii), an assumption $\lambda^{* \tau} \neq \lambda^{\tau}$ lead to a contradiction. Therefore we have $\lambda^{* \tau}=\lambda^{\tau}$.

Finally, if $\lambda^{\tau}=(N-M)^{\tau}$ or $M^{\tau}$, then we must have only the case (b), so $\lambda$ is a $F$-point of $M(L)_{>0}$.

ReMARK. $F\left(\lambda_{(3)}\right)<1 \Leftrightarrow \exists c \in \mathbf{Z} ; F(c+\lambda)<1$.

Corollary 3.7. Let $\mathscr{R}$ be a reduced lattice with basis $\{1, \lambda, \mu\}$ such that $F(\mu)>1, \quad 2\left|Y_{\mu}\right|<1, \quad 0<X_{\mu}<X_{\lambda}, \quad 0<\omega_{1}(\lambda, \mu)<1, \quad F\left(\lambda_{(3)}\right)<1 . \quad$ If $\theta_{g}=$ $x+y \lambda+z \mu(x, y, z \in \mathbf{Z})$, then $(y, z) \in\{(1,0),(1,1),(1,-1),(2,1),(d, 1),(d+1,1)$, $(2 d+1,2),(d-1,1),(3 d+2,3)\}$, where $d=\left[1 / \omega_{1}(\lambda, \mu)\right] \geq 1$.

\section{Preliminaries (I)}

Definition 4.1. Let $\mathscr{R}$ be a lattice of $K$. For a basis $\{1, \lambda, \mu\}$ of $\mathscr{R}$, we define a mapping $F_{\lambda, \mu}: \mathbf{R}^{3} \rightarrow \mathbf{R}$ by $F_{\lambda, \mu}(x, y, z)=x^{2}+\left(\lambda^{\prime}+\lambda^{\prime \prime}\right) x y+\left(\mu^{\prime}+\mu^{\prime \prime}\right) x z$ $+\left(\lambda^{\prime} \mu^{\prime \prime}+\lambda^{\prime \prime} \mu^{\prime}\right) y z+\lambda^{\prime} \lambda^{\prime \prime} y^{2}+\mu^{\prime} \mu^{\prime \prime} z^{2}$. For any $(x, y, z) \in \mathbf{Z}^{3}$, we have $F_{\lambda, \mu}(x, y, z)$ $=F(x+y \lambda+z \mu)$.

REMARK. $\quad F_{\lambda, \mu}$ is a positive quadratic form with real coefficients of rank 2 . $\left(\omega_{2}, 1, \omega_{1}\right)$ is an isotropic vector of $F_{\lambda, \mu}$. 
We quote Lahlou and Farhane [5], Lemma 2.2 as Lemma 4.2 for our convenience. (cf. [1], Lemma 2.2)

Lemma 4.2 (Lahlou and Farhane [5], Lemma 2.2). Let $\mathscr{R}$ be a lattice of $K$ and let $\{1, \lambda, \mu\}$ be a basis of $\mathscr{R}$. Then we can write

(1) $F_{\lambda, \mu}(x, y, z)=a\left(z-\omega_{1} y\right)^{2}+2 b\left(z-\omega_{1} y\right)\left(x-\omega_{2} y\right)+\left(x-\omega_{2} y\right)^{2}$

$$
F_{\lambda, \mu}(x, y, z)=\frac{1}{2}\left(x-\omega_{2} y\right)^{2}+\frac{1}{2}\left(x-\omega_{2} y+2 b\left(z-\omega_{1} y\right)\right)^{2}+\left(a-2 b^{2}\right)\left(z-\omega_{1} y\right)^{2}
$$

$$
F_{\lambda, \mu}(x, y, z)=\frac{a}{2}\left(z-\omega_{1} y\right)^{2}+\frac{a}{2}\left(z-\omega_{1} y+\frac{2 b}{a}\left(x-\omega_{2} y\right)\right)^{2}+\left(1-\frac{2 b^{2}}{a}\right)\left(x-\omega_{2} y\right)^{2}
$$

with $a=F(\mu), b=Y_{\mu}$.

Definition 4.3. Let $\mathscr{R}$ be a reduced lattice with basis $\{1, \lambda, \mu\}$ such that $\mu>-1 / 2, \omega_{2}(\lambda, \mu)>0,0<\omega_{1}(\lambda, \mu)<1$. Let $y \in \mathbf{Z}$. Then we define

$$
\begin{aligned}
& \psi_{1, y}=\left[\omega_{2} y\right]-1+y \lambda+\left[\omega_{1} y\right] \mu \quad \psi_{7, y}=\left[\omega_{2} y\right]+1+y \lambda+\left(\left[\omega_{1} y\right]-1\right) \mu \\
& \psi_{2, y}=\left[\omega_{2} y\right]-1+y \lambda+\left(\left[\omega_{1} y\right]+1\right) \mu \quad \psi_{8, y}=\left[\omega_{2} y\right]+1+y \lambda+\left[\omega_{1} y\right] \mu \\
& \psi_{3, y}=\left[\omega_{2} y\right]+y \lambda+\left(\left[\omega_{1} y\right]-1\right) \mu \quad \psi_{9, y}=\left[\omega_{2} y\right]+1+y \lambda+\left(\left[\omega_{1} y\right]+1\right) \mu \\
& \psi_{4, y}=\left[\omega_{2} y\right]+y \lambda+\left[\omega_{1} y\right] \mu \quad \psi_{10, y}=\left[\omega_{2} y\right]+1+y \lambda+\left(\left[\omega_{1} y\right]+2\right) \mu \\
& \psi_{5, y}=\left[\omega_{2} y\right]+y \lambda+\left(\left[\omega_{1} y\right]+1\right) \mu \quad \psi_{11, y}=\left[\omega_{2} y\right]+2+y \lambda+\left[\omega_{1} y\right] \mu \\
& \psi_{6, y}=\left[\omega_{2} y\right]+y \lambda+\left(\left[\omega_{1} y\right]+2\right) \mu \quad \psi_{12, y}=\left[\omega_{2} y\right]+2+y \lambda+\left(\left[\omega_{1} y\right]+1\right) \mu \\
& \phi_{1}=\psi_{4,1}=\left[\omega_{2}\right]+\lambda \quad \phi_{5}=\psi_{2,1}=\left[\omega_{2}\right]-1+\lambda+\mu \quad \phi_{9}=2 \lambda+\mu \\
& \phi_{2}=\psi_{5,1}=\left[\omega_{2}\right]+\lambda+\mu \quad \phi_{6}=\psi_{8,1}=\left[\omega_{2}\right]+1+\lambda \quad \phi_{10}=3 \lambda+2 \mu \\
& \phi_{3}=\psi_{3,1}=\left[\omega_{2}\right]+\lambda-\mu \quad \phi_{7}=\psi_{7,1}=\left[\omega_{2}\right]+1+\lambda-\mu \\
& \phi_{4}=\psi_{1,1}=\left[\omega_{2}\right]-1+\lambda \quad \phi_{8}=\psi_{9,1}=\left[\omega_{2}\right]+1+\lambda+\mu
\end{aligned}
$$

REMARK 4.4. (1) If $0<\mu<1$, then we have 


$$
\begin{aligned}
& \psi_{1, y}<\psi_{2, y}<\psi_{4, y} ; \quad \psi_{1, y}<\psi_{3, y}<\psi_{4, y} ; \quad \psi_{4, y}<\psi_{5, y}<\psi_{6, y}<\psi_{9, y} \\
& \psi_{4, y}<\psi_{5, y}<\psi_{8, y}<\psi_{9, y} ; \quad \psi_{4, y}<\psi_{7, y}<\psi_{8, y}<\psi_{9, y} \\
& \psi_{9, y}<\psi_{10, y}<\psi_{12, y} ; \quad \psi_{9, y}<\psi_{11, y}<\psi_{12, y}
\end{aligned}
$$

(2) If $\mu>1$, then we have

$$
\begin{aligned}
& \psi_{3, y}<\psi_{1, y}<\psi_{4, y} ; \quad \psi_{3, y}<\psi_{7, y}<\psi_{4, y} ; \quad \psi_{4, y}<\psi_{2, y}<\psi_{5, y}<\psi_{9, y} \\
& \psi_{4, y}<\psi_{8, y}<\psi_{5, y}<\psi_{9, y} ; \quad \psi_{4, y}<\psi_{8, y}<\psi_{11, y}<\psi_{9, y} \\
& \psi_{9, y}<\psi_{6, y}<\psi_{10, y} ; \quad \psi_{9, y}<\psi_{12, y}<\psi_{10, y}
\end{aligned}
$$

Lemma 4.5. Let $\mathscr{R}$ be a reduced lattice with basis $\{1, \lambda, \mu\}$ such that $\mu>-1 / 2, \omega_{2}(\lambda, \mu)>0$ and $0<\omega_{1}(\lambda, \mu)<1$. Let $a>\max \left(1,2 b^{2}, 2|b|\right)$, where $a=F(\mu), b=Y_{\mu}$. Then

(1) $\theta_{g} \in\left\{\psi_{i, y} ; y(\neq 0) \in \mathbf{Z}, 1 \leq i \leq 12\right\}$.

(2) $\lambda, \mu>0 \Rightarrow \psi_{i, 1} \leq \psi_{i, y}(y \geq 1)$.

(3) (i) $b<0 \Rightarrow F\left(\psi_{2, y}\right)>1, F\left(\psi_{6, y}\right)>1, F\left(\psi_{7, y}\right)>1, F\left(\psi_{11, y}\right)>1$.

(ii) $b>0 \Rightarrow F\left(\psi_{1, y}\right)>1, F\left(\psi_{3, y}\right)>1, F\left(\psi_{10, y}\right)>1, F\left(\psi_{12, y}\right)>1$.

(4) $F\left(\psi_{3,1}\right)>F\left(\psi_{4,1}\right)$.

(5) $(0<) b<1 / 2 \Rightarrow F\left(\psi_{7,1}\right)>F\left(\psi_{4,1}\right)$.

(6) $F\left(\psi_{5,1}\right)<F\left(\psi_{4,1}\right), 0<b<1 \Rightarrow F\left(\psi_{7,1}\right)>F\left(\psi_{4,1}\right)$.

(7) $b>1 \Rightarrow F\left(\psi_{7,1}\right)>1$.

(8) $b>0$ or $-1 / 2<b<0 \Rightarrow F\left(\psi_{1,1}\right)>F\left(\psi_{4,1}\right)$.

(9) $F\left(\psi_{5,1}\right)>F\left(\psi_{8,1}\right),(0<) b<1 \Rightarrow F\left(\psi_{2,1}\right)>F\left(\psi_{4,1}\right)$.

(10) $F\left(\psi_{4,1}\right)>F\left(\psi_{8,1}\right), b<0 \Rightarrow c_{2}=\left[\omega_{2}\right]-\omega_{2}<-1 / 2$.

(11) $c_{1}=\left[\omega_{1}\right]-\omega_{1}<-1 / 2, b<0 \Rightarrow F\left(\psi_{8,1}\right)>F\left(\psi_{9,1}\right)$.

(12) $[2 \alpha]=\left\{\begin{array}{ll}2[\alpha] & \text { if } 0 \leq \alpha-[\alpha]<1 / 2 \\ 2[\alpha]+1 & \text { if } 1 / 2 \leq \alpha-[\alpha]\end{array}\right.$.

Proof. We put $c_{1}=\left[\omega_{1}\right]-\omega_{1}, c_{2}=\left[\omega_{2}\right]-\omega_{2}$. Then $-1<c_{1}, c_{2}<0$.

(1) was proved in Lahlou and Farhane [5], Theorem 2.1.

(2) obvious

(3) by Lemma 4.2,(1)

(4) By Lemma 4.2,(1), $F\left(\psi_{3,1}\right)-F\left(\psi_{4,1}\right)=-2 a c_{1}+a-2 b c_{2}=-2 a c_{1}+$ $a\left(1-\frac{2 b}{a} c_{2}\right)>0$. 
(5) By Lemma 4.2,(1), $\quad F\left(\psi_{7,1}\right)-F\left(\psi_{4,1}\right)=-2 a c_{1}+a+2 b c_{1}-2 b c_{2}-2 b+$ $2 c_{2}+1=(1-2 b)\left(1+c_{2}\right)+a+c_{2}-2(a-b) c_{1}>0$.

(6) By Lemma 4.2,(1) since $F\left(\psi_{5,1}\right)<F\left(\psi_{4,1}\right), F\left(\psi_{4,1}\right)-F\left(\psi_{5,1}\right)=-2 a c_{1}-$ $a-2 b c_{2}>0$. So $-2 b c_{2}>a\left(1+2 c_{1}\right)$. From this and $a>2 b$, we have $-2 b c_{2}>$ $2 b\left(1+2 c_{1}\right),-c_{2}>1+2 c_{1}$. Hence $-2 c_{1}>1+c_{2}$. By this,

$$
\begin{aligned}
F\left(\psi_{7,1}\right)-F\left(\psi_{4,1}\right) & =-2 a c_{1}+a+2 b c_{1}-2 b c_{2}-2 b+2 c_{2}+1 \\
& =(1-2 b)\left(1+c_{2}\right)+a+c_{2}-2 c_{1}(a-b) \\
& >(1-2 b)\left(1+c_{2}\right)+a+c_{2}+\left(1+c_{2}\right)(a-b) \\
& =(1-2 b)\left(1+c_{2}\right)+a-1+1+c_{2}+\left(1+c_{2}\right)(a-b) \\
& =(2-2 b)\left(1+c_{2}\right)+a-1+\left(1+c_{2}\right)(a-b)>0 .
\end{aligned}
$$

(7) If $b>1$, then we have $a>2$ because $a>2|b|$. From this and by Lemma 4.2,(3), we have $F\left(\psi_{7,1}\right)>1$.

(8) By Lemma 4.2,(1), $F\left(\psi_{1,1}\right)-F\left(\psi_{4,1}\right)=-2 b c_{1}-2 c_{2}+1>0$.

(9) Since $F\left(\psi_{5,1}\right)>F\left(\psi_{8,1}\right)$, we have $F\left(\psi_{5,1}\right)-F\left(\psi_{8,1}\right)=2 a c_{1}+a+2 b c_{2}-$ $2 b c_{1}-2 c_{2}-1>0$. From this, $F\left(\psi_{2,1}\right)-F\left(\psi_{4,1}\right)=2 a c_{1}+a-2 b c_{1}+2 b c_{2}-2 b-$ $2 c_{2}+1=\left(2 a c_{1}+a+2 b c_{2}-2 b c_{1}-2 c_{2}-1\right)+2-2 b>0$.

(10) Since $F\left(\psi_{4,1}\right)-F\left(\psi_{8,1}\right)>0$, we have $b c_{1}+c_{2}<-1 / 2$. From this and $b<0, c_{1}<0$, we have $c_{2}<-1 / 2$.

(11) By Lemma 4.2,(1), $F\left(\psi_{9,1}\right)-F\left(\psi_{8,1}\right)=2 a c_{1}+a+2 b\left(c_{2}+1\right)=$ $a\left(2 c_{1}+1\right)+2 b\left(c_{2}+1\right)<0$.

(12) is easily deduced from the definitions.

Some of Lemma 4.5 were proved in Lahlou and Farhane [5], Theorem 2.1 .

REMARK. $\quad a>1,2|b|<1 \Rightarrow a>\max \left(1,4 b^{2}\right) \Rightarrow a>\max \left(1,2 b^{2}, 2|b|\right)$.

\section{Preliminaries (II)}

In this section, we make the following assumption;

Assumption 5.1. Let $\mathscr{R}=\langle 1, \lambda, \mu\rangle$ be a reduced lattice of $K$ such that (a) $0<\lambda<1,-1 / 2<\mu, F(\mu)>1,2\left|Y_{\mu}\right|<1,0<X_{\mu}<X_{\lambda}, 0<\omega_{1}(\lambda, \mu)<1$

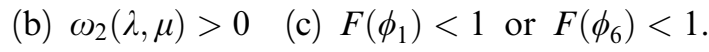


By Theorem 2.10, we can take such the basis. So in next section, we shall consider six cases:

$$
\begin{array}{ll}
(1 A) 0<\mu<1, \phi_{1}>1 & (2 A) \mu>1, \phi_{1}>1 \\
(3 A) \mu<0, \phi_{1}>1 & \\
(1 B) 0<\mu<1, \phi_{1}<1, F\left(\phi_{6}\right)<1 & (2 B) \mu>1, \phi_{1}<1, F\left(\phi_{6}\right)<1 \\
\text { (3B) } \mu<0, \phi_{1}<1, F\left(\phi_{6}\right)<1 &
\end{array}
$$

We note that
(A) $\phi_{1}=\left[\omega_{2}\right]+\lambda>1 \Leftrightarrow\left[\omega_{2}\right] \geq 1 \Leftrightarrow \omega_{2}>1$,
(B) $\phi_{1}=\left[\omega_{2}\right]+\lambda<1 \Leftrightarrow\left[\omega_{2}\right]=0 \Leftrightarrow \omega_{2}<1$.

LEMMA 5.2. If $\phi_{1}<1$, then

(1) $Y_{\lambda}<-1 / 2 \quad$ (2) $\omega_{2}(\lambda, \mu)>1 / 2-\omega_{1} Y_{\mu}$.

Proof. (1) From $\phi_{1}=\left[\omega_{2}\right]+\lambda<1$, we have $\left[\omega_{2}\right]=0$. By definition $\lambda_{(1)}=$ $\left[-Y_{\lambda}\right]+\lambda, \quad \lambda_{(2)}=\left[-Y_{\lambda}\right]+1+\lambda$. Since $\mathscr{R}$ is a reduced lattice, from $\phi_{1}<1$, we have $F\left(\phi_{1}\right)>1$. Hence, by Assumpsion 5.1,(c), we have $F\left(\phi_{6}\right)<1$. From $F\left(\phi_{6}\right)=F\left(\left[\omega_{2}\right]+1+\lambda\right)=F(1+\lambda)<1$, we have $1+\lambda=\lambda_{(1)}$ or $\lambda_{(2)}$.

(i) The case $1+\lambda=\lambda_{(1)}$. Since $-1<Y_{\lambda}+1=Y_{\lambda_{(1)}}<0$, we have $-2<Y_{\lambda}<$ -1 .

(ii) The case $1+\lambda=\lambda_{(2)}$. We have $\lambda=\lambda_{(1)}$. Since $F\left(\lambda_{(2)}\right)<1$, we have $0<Y_{\lambda_{(2)}}<1 / 2$. From this, $0<Y_{\lambda}+1=Y_{\lambda_{(2)}}<1 / 2$, so $-1<Y_{\lambda}<-1 / 2$.

Finally, from (i)(ii), we have $Y_{\lambda}<-1 / 2$.

(2) From (1), we have $-Y_{\lambda}>1 / 2$. Hence, $\omega_{2}(\lambda, \mu)=-Y_{\lambda}-\omega_{1} Y_{\mu}>$ $1 / 2-\omega_{1} Y_{\mu}$.

COROLlary 5.3. $Y_{\mu}<0 \Rightarrow \omega_{2}(\lambda, \mu)>1 / 2$.

By Corollary 3.7 if $\theta_{g}=x+y \lambda+z \mu(x, y, z \in \mathbf{Z})$, then $(y, z) \in\{(1,0)$, $(1,1),(1,-1),(2,1),(d, 1),(d+1,1),(2 d+1,2),(d-1,1),(3 d+2,3)\}$, where $d=$ $\left[1 / \omega_{1}(\lambda, \mu)\right] \geq 1$.

From Remark 3.5 and Corollary 5.3, we make the following tables in which we deside whether the possibility that $\theta_{g}=\psi_{i, y}(1 \leq i \leq 10, i=12)$ exists. Note that $y \geq 1 \Rightarrow\left[y \omega_{2}\right] \geq y\left[\omega_{2}\right]$. 
Table 1

\begin{tabular}{|l|l|l|l|l|l|l|}
\hline \multicolumn{1}{|c|}{$(y, z)$} & \multicolumn{1}{|c|}{$\begin{array}{c}\psi_{1, y}=\left[\omega_{2} y\right]-1+y \lambda \\
+\left[\omega_{1} y\right] \mu\end{array}$} & $\begin{array}{c}\mu>0 \\
\omega_{2}>1\end{array}$ & $\begin{array}{c}\mu<0 \\
\omega_{2}>1\end{array}$ & $\begin{array}{c}\mu>0 \\
\omega_{2}<1\end{array}$ & $\begin{array}{c}\mu<0 \\
\omega_{2}<1\end{array}$ & No. \\
\hline \hline$(1,0)$ & {$\left[\omega_{2}\right]-1+\lambda$} & & & $<1$ & $<1$ & $(1-1)$ \\
\hline$(1,1)$ & {$\left[\omega_{2}\right]-1+\lambda$} & impossible & impossible & impossible & impossible & \\
\hline$(1,-1)$ & {$\left[\omega_{2}\right]-1+\lambda$} & impossible & impossible & impossible & impossible & \\
\hline$(2,1)$ & {$\left[2 \omega_{2}\right]-1+2 \lambda+\left[2 \omega_{1}\right] \mu$} & & & & & $(1-2)$ \\
\hline$(d, 1)$ & {$\left[d \omega_{2}\right]-1+d \lambda$} & impossible & impossible & impossible & impossible & \\
\hline$(d+1,1)$ & {$\left[(d+1) \omega_{2}\right]-1+(d+1) \lambda+\mu$} & & & & & $(1-3)$ \\
\hline$(2 d+1,2)$ & $\begin{array}{c}{\left[(2 d+1) \omega_{2}\right]-1+(2 d+1) \lambda} \\
+\left[(2 d+1) \omega_{1}\right] \mu\end{array}$ & $>\phi_{6}$ & & & & $(1-4)$ \\
\hline$(d-1,1)$ & {$\left[(d-1) \omega_{2}\right]-1+(d-1) \lambda$} & impossible & impossible & impossible & impossible & \\
\hline$(3 d+2,3)$ & $\begin{array}{c}{\left[(3 d+2) \omega_{2}\right]-1+(3 d+2) \lambda} \\
+\left[(3 d+2) \omega_{1}\right] \mu\end{array}$ & $>\phi_{6}$ & $>\phi_{6}$ & & & $(1-5)$ \\
\hline
\end{tabular}

Table 2. $(\mu>0)$

\begin{tabular}{|l|l|l|l|l|}
\hline$(y, z)$ & $\psi_{2, y}=\left[\omega_{2} y\right]-1+y \lambda+\left(\left[\omega_{1} y\right]+1\right) \mu$ & $\begin{array}{c}\mu>0 \\
\omega_{2}>1\end{array}$ & $\begin{array}{c}\mu>0 \\
\omega_{2}<1\end{array}$ & No. \\
\hline \hline$(1,0)$ & {$\left[\omega_{2}\right]-1+\lambda+\mu$} & impossible & impossible & \\
\hline$(1,1)$ & {$\left[\omega_{2}\right]-1+\lambda+\mu$} & & & $(2-1)$ \\
\hline$(1,-1)$ & {$\left[\omega_{2}\right]-1+\lambda+\mu$} & impossible & impossible & \\
\hline$(2,1)$ & {$\left[2 \omega_{2}\right]-1+2 \lambda+\left(\left[2 \omega_{1}\right]+1\right) \mu$} & & $(2-2)$ \\
\hline$(d, 1)$ & {$\left[d \omega_{2}\right]-1+d \lambda+\mu$} & & $(2-3)$ \\
\hline$(d+1,1)$ & {$\left[(d+1) \omega_{2}\right]-1+(d+1) \lambda+2 \mu$} & impossible & impossible & \\
\hline$(2 d+1,2)$ & {$\left[(2 d+1) \omega_{2}\right]-1+(2 d+1) \lambda$} & $>\phi_{6}$ & & $(2-4)$ \\
\hline$(d-1,1)$ & {$\left[(d-1) \omega_{2}\right]-1+(d-1) \lambda+\mu$} & & & $(2-5)$ \\
\hline$(3 d+2,3)$ & {$\left[(3 d+2) \omega_{2}\right]-1+(3 d+2) \lambda$} & & & $(2-6)$ \\
\hline
\end{tabular}


Table 3

\begin{tabular}{|l|l|l|l|l|l|l|}
\hline \multicolumn{1}{|c|}{$(y, z)$} & \multicolumn{1}{|c|}{$\begin{array}{c}\psi_{3, y}=\left[\omega_{2} y\right]+y \lambda \\
+\left(\left[\omega_{1} y\right]-1\right) \mu\end{array}$} & $\begin{array}{c}\mu>0 \\
\omega_{2}>1\end{array}$ & $\begin{array}{c}\mu<0 \\
\omega_{2}>1\end{array}$ & $\begin{array}{c}\mu>0 \\
\omega_{2}<1\end{array}$ & $\begin{array}{c}\mu<0 \\
\omega_{2}<1\end{array}$ & No. \\
\hline \hline$(1,0)$ & {$\left[\omega_{2}\right]+\lambda-\mu$} & impossible & impossible & impossible & impossible & \\
\hline$(1,1)$ & {$\left[\omega_{2}\right]+\lambda-\mu$} & impossible & impossible & impossible & impossible & \\
\hline$(1,-1)$ & {$\left[\omega_{2}\right]+\lambda-\mu$} & & & $<1$ & & $(3-1)$ \\
\hline$(2,1)$ & {$\left[2 \omega_{2}\right]+2 \lambda+\left(\left[2 \omega_{1}\right]-1\right) \mu$} & impossible & impossible & impossible & impossible & \\
\hline$(d, 1)$ & {$\left[d \omega_{2}\right]+d \lambda-\mu$} & impossible & impossible & impossible & impossible & \\
\hline$(d+1,1)$ & {$\left[(d+1) \omega_{2}\right]+(d+1) \lambda$} & impossible & impossible & impossible & impossible & \\
\hline$(2 d+1,2)$ & {$\left[(2 d+1) \omega_{2}\right]+(2 d+1) \lambda$} \\
$+\left(\left[(2 d+1) \omega_{1}\right]-1\right) \mu$ & impossible & impossible & impossible & impossible & \\
\hline$(d-1,1)$ & {$\left[(d-1) \omega_{2}\right]+(d-1) \lambda-\mu$} & impossible & impossible & impossible & impossible & \\
\hline$(3 d+2,3)$ & {$\left[(3 d+2) \omega_{2}\right]+(3 d+2) \lambda$} \\
$+\left(\left[(3 d+2) \omega_{1}\right]-1\right) \mu$ & $>\phi_{6}$ & $>\phi_{6}$ & & & $(3-2)$ \\
\hline
\end{tabular}

Table 4

\begin{tabular}{|l|l|l|l|l|l|l|}
\hline \multicolumn{1}{|c|}{$(y, z)$} & $\psi_{4, y}=\left[\omega_{2} y\right]+y \lambda+\left[\omega_{1} y\right] \mu$ & $\begin{array}{c}\mu>0 \\
\omega_{2}>1\end{array}$ & $\begin{array}{c}\mu<0 \\
\omega_{2}>1\end{array}$ & $\begin{array}{c}\mu>0 \\
\omega_{2}<1\end{array}$ & $\begin{array}{c}\mu<0 \\
\omega_{2}<1\end{array}$ & No. \\
\hline \hline$(1,0)$ & {$\left[\omega_{2}\right]+\lambda$} & & & $<1$ & $<1$ & $(4-1)$ \\
\hline$(1,1)$ & {$\left[\omega_{2}\right]+\lambda$} & impossible & impossible & impossible & impossible & \\
\hline$(1,-1)$ & {$\left[\omega_{2}\right]+\lambda$} & impossible & impossible & impossible & impossible & \\
\hline$(2,1)$ & {$\left[2 \omega_{2}\right]+2 \lambda+\left[2 \omega_{1}\right] \mu$} & $>\phi_{6}$ & & & & $(4-2)$ \\
\hline$(d, 1)$ & {$\left[d \omega_{2}\right]+d \lambda$} & impossible & impossible & impossible & impossible & \\
\hline$(d+1,1)$ & {$\left[(d+1) \omega_{2}\right]+(d+1) \lambda+\mu$} & $>\phi_{6}$ & & & & $(4-3)$ \\
\hline$(2 d+1,2)$ & {$\left[(2 d+1) \omega_{2}\right]+(2 d+1) \lambda$} & $>\phi_{6}$ & $>\phi_{6}$ & & & $(4-4)$ \\
\hline$(d-1,1)$ & {$\left[(d-1) \omega_{2}\right]+(d-1) \lambda$} & impossible & impossible & impossible & impossible & \\
\hline$(3 d+2,3)$ & {$\left[(3 d+2) \omega_{2}\right]+(3 d+2) \lambda$} & $>\phi_{6}$ & $>\phi_{6}$ & & & $(4-5)$ \\
\hline
\end{tabular}


Table 5

\begin{tabular}{|l|l|l|l|l|l|l|}
\hline \multicolumn{1}{|c|}{$(y, z)$} & \multicolumn{1}{|c|}{$\begin{array}{c}\psi_{5, y}=\left[\omega_{2} y\right]+y \lambda \\
+\left(\left[\omega_{1} y\right]+1\right) \mu\end{array}$} & $\begin{array}{c}\mu>0 \\
\omega_{2}>1\end{array}$ & $\begin{array}{c}\mu<0 \\
\omega_{2}>1\end{array}$ & $\begin{array}{c}\mu>0 \\
\omega_{2}<1\end{array}$ & $\begin{array}{c}\mu<0 \\
\omega_{2}<1\end{array}$ & No. \\
\hline \hline$(1,0)$ & {$\left[\omega_{2}\right]+\lambda+\mu$} & impossible & impossible & impossible & impossible & \\
\hline$(1,1)$ & {$\left[\omega_{2}\right]+\lambda+\mu$} & & & $<1$ & $(5-1)$ \\
\hline$(1,-1)$ & {$\left[\omega_{2}\right]+\lambda+\mu$} & impossible & impossible & impossible & impossible & \\
\hline$(2,1)$ & {$\left[2 \omega_{2}\right]+2 \lambda+\left(\left[2 \omega_{1}\right]+1\right) \mu$} & $>\phi_{6}$ & & & $(5-2)$ \\
\hline$(d, 1)$ & {$\left[d \omega_{2}\right]+d \lambda+\mu$} & $>\phi_{6}(d \geq 2)$ & & & & $(5-3)$ \\
\hline$(d+1,1)$ & {$\left[(d+1) \omega_{2}\right]+(d+1) \lambda+2 \mu$} & impossible & impossible & impossible & impossible & \\
\hline$(2 d+1,2)$ & $\begin{array}{c}{\left[(2 d+1) \omega_{2}\right]+(2 d+1) \lambda} \\
+\left(\left[(2 d+1) \omega_{1}\right]+1\right) \mu\end{array}$ & $>\phi_{6}$ & $>\phi_{6}$ & & & $(5-4)$ \\
\hline$(d-1,1)$ & {$\left[(d-1) \omega_{2}\right]+(d-1) \lambda+\mu$} & $>\phi_{6}(d \geq 3)$ & & & & $(5-5)$ \\
\hline$(3 d+2,3)$ & $\begin{array}{c}{\left[(3 d+2) \omega_{2}\right]+(3 d+2) \lambda} \\
+\left(\left[(3 d+2) \omega_{1}\right]+1\right) \mu\end{array}$ & $>\phi_{6}$ & $>\phi_{6}$ & & & $(5-6)$ \\
\hline
\end{tabular}

Table 6. $(\mu>0)$

\begin{tabular}{|l|l|l|l|}
\hline$(y, z)$ & \multicolumn{1}{|c|}{$\psi_{6, y}=\left[\omega_{2} y\right]+y \lambda+\left(\left[\omega_{1} y\right]+2\right) \mu$} & $\begin{array}{c}\mu>0 \\
\omega_{2} \lessgtr 1\end{array}$ & No. \\
\hline \hline$(1,0)$ & {$\left[\omega_{2}\right]+\lambda+2 \mu$} & impossible & \\
\hline$(1,1)$ & {$\left[\omega_{2}\right]+\lambda+2 \mu$} & impossible & \\
\hline$(1,-1)$ & {$\left[\omega_{2}\right]+\lambda+2 \mu$} & impossible & \\
\hline$(2,1)$ & {$\left[2 \omega_{2}\right]+2 \lambda+\left(\left[2 \omega_{1}\right]+2\right) \mu$} & impossible & \\
\hline$(d, 1)$ & {$\left[d \omega_{2}\right]+d \lambda+2 \mu$} & impossible & \\
\hline$(d+1,1)$ & {$\left[(d+1) \omega_{2}\right]+(d+1) \lambda+3 \mu$} & impossible & \\
\hline$(2 d+1,2)$ & {$\left[(2 d+1) \omega_{2}\right]+(2 d+1) \lambda+\left(\left[(2 d+1) \omega_{1}\right]+2\right) \mu$} & impossible & \\
\hline$(d-1,1)$ & {$\left[(d-1) \omega_{2}\right]+(d-1) \lambda+2 \mu$} & impossible & \\
\hline$(3 d+2,3)$ & {$\left[(3 d+2) \omega_{2}\right]+(3 d+2) \lambda+\left(\left[(3 d+2) \omega_{1}\right]+2\right) \mu$} & impossible & \\
\hline
\end{tabular}


Table 7. $(\mu>0)$

\begin{tabular}{|l|l|l|l|}
\hline \multicolumn{1}{|c|}{$(y, z)$} & \multicolumn{1}{|c|}{$\psi_{7, y}=\left[\omega_{2} y\right]+1+y \lambda+\left(\left[\omega_{1} y\right]-1\right) \mu$} & $\begin{array}{c}\mu>0 \\
\omega_{2} \lessgtr 1\end{array}$ & No. \\
\hline \hline$(1,0)$ & {$\left[\omega_{2}\right]+1+\lambda-\mu$} & impossible & \\
\hline$(1,1)$ & {$\left[\omega_{2}\right]+1+\lambda-\mu$} & impossible & \\
\hline$(1,-1)$ & {$\left[\omega_{2}\right]+1+\lambda-\mu$} & & $(7-1)$ \\
\hline$(2,1)$ & {$\left[2 \omega_{2}\right]+1+2 \lambda+\left(\left[2 \omega_{1}\right]-1\right) \mu$} & impossible & \\
\hline$(d, 1)$ & {$\left[d \omega_{2}\right]+1+d \lambda-\mu$} & impossible & \\
\hline$(d+1,1)$ & {$\left[(d+1) \omega_{2}\right]+1+(d+1) \lambda$} & impossible & \\
\hline$(2 d+1,2)$ & {$\left[(2 d+1) \omega_{2}\right]+1+(2 d+1) \lambda+\left(\left[(2 d+1) \omega_{1}\right]-1\right) \mu$} & impossible & \\
\hline$(d-1,1)$ & {$\left[(d-1) \omega_{2}\right]+1+(d-1) \lambda-\mu$} & impossible & \\
\hline$(3 d+2,3)$ & {$\left[(3 d+2) \omega_{2}\right]+1+(3 d+2) \lambda+\left(\left[(3 d+2) \omega_{1}\right]-1\right) \mu$} & $>\phi_{6}$ & \\
\hline
\end{tabular}

Table 8

\begin{tabular}{|l|l|l|l|}
\hline$(y, z)$ & \multicolumn{1}{|c|}{$\psi_{8, y}=\left[\omega_{2} y\right]+1+y \lambda+\left[\omega_{1} y\right] \mu$} & $\begin{array}{c}\mu \lessgtr 0 \\
\omega_{2} \lessgtr 1\end{array}$ & No. \\
\hline \hline$(1,0)$ & {$\left[\omega_{2}\right]+1+\lambda$} & & $(8-1)$ \\
\hline$(1,1)$ & {$\left[\omega_{2}\right]+1+\lambda$} & impossible & \\
\hline$(1,-1)$ & {$\left[\omega_{2}\right]+1+\lambda$} & impossible & \\
\hline$(2,1)$ & {$\left[2 \omega_{2}\right]+1+2 \lambda+\left[2 \omega_{1}\right] \mu$} & $>\phi_{6}$ & \\
\hline$(d, 1)$ & {$\left[d \omega_{2}\right]+1+d \lambda$} & impossible & \\
\hline$(d+1,1)$ & {$\left[(d+1) \omega_{2}\right]+1+(d+1) \lambda+\mu$} & $>\phi_{6}$ & \\
\hline$(2 d+1,2)$ & {$\left[(2 d+1) \omega_{2}\right]+1+(2 d+1) \lambda+\left[(2 d+1) \omega_{1}\right] \mu$} & $>\phi_{6}$ & \\
\hline$(d-1,1)$ & {$\left[(d-1) \omega_{2}\right]+1+(d-1) \lambda$} & impossible & \\
\hline$(3 d+2,3)$ & {$\left[(3 d+2) \omega_{2}\right]+1+(3 d+2) \lambda+\left[(3 d+2) \omega_{1}\right] \mu$} & $>\phi_{6}$ & \\
\hline
\end{tabular}


Table 9. $(\mu<0)$

\begin{tabular}{|l|l|l|l|}
\hline \multicolumn{1}{|c|}{$(y, z)$} & \multicolumn{1}{|c|}{$\psi_{9, y}=\left[\omega_{2} y\right]+1+y \lambda+\left(\left[\omega_{1} y\right]+1\right) \mu$} & $\begin{array}{c}\mu<0 \\
\omega_{2} \lessgtr 1\end{array}$ & No. \\
\hline \hline$(1,0)$ & {$\left[\omega_{2}\right]+1+\lambda+\mu$} & impossible & \\
\hline$(1,1)$ & {$\left[\omega_{2}\right]+1+\lambda+\mu$} & & $(9-1)$ \\
\hline$(1,-1)$ & {$\left[\omega_{2}\right]+1+\lambda+\mu$} & impossible & \\
\hline$(2,1)$ & {$\left[2 \omega_{2}\right]+1+2 \lambda+\left(\left[2 \omega_{1}\right]+1\right) \mu$} & $>\phi_{6}$ & \\
\hline$(d, 1)$ & {$\left[d \omega_{2}\right]+1+d \lambda+\mu$} & $>\phi_{6}(d \geq 2)$ & \\
\hline$(d+1,1)$ & {$\left[(d+1) \omega_{2}\right]+1+(d+1) \lambda+2 \mu$} & impossible & \\
\hline$(2 d+1,2)$ & {$\left[(2 d+1) \omega_{2}\right]+1+(2 d+1) \lambda+\left(\left[(2 d+1) \omega_{1}\right]+1\right) \mu$} & $>\phi_{6}$ & \\
\hline$(d-1,1)$ & {$\left[(d-1) \omega_{2}\right]+1+(d-1) \lambda+\mu$} & $>\phi_{6}(d \geq 3)$ & \\
\hline$(3 d+2,3)$ & {$\left[(3 d+2) \omega_{2}\right]+1+(3 d+2) \lambda+\left(\left[(3 d+2) \omega_{1}\right]+1\right) \mu$} & $>\phi_{6}$ & \\
\hline
\end{tabular}

Table 10. $(\mu<0)$

\begin{tabular}{|l|l|l|l|}
\hline$(y, z)$ & \multicolumn{1}{|c|}{$\psi_{10, y}=\left[\omega_{2} y\right]+1+y \lambda+\left(\left[\omega_{1} y\right]+2\right) \mu$} & \multicolumn{1}{c|}{$\begin{array}{c}\omega_{2} \lessgtr 1 \\
\text { No }\end{array}$} & No. \\
\hline \hline$(1,0)$ & {$\left[\omega_{2}\right]+1+\lambda+2 \mu$} & impossible & \\
\hline$(1,1)$ & {$\left[\omega_{2}\right]+1+\lambda+2 \mu$} & impossible & \\
\hline$(1,-1)$ & {$\left[\omega_{2}\right]+1+\lambda+2 \mu$} & impossible & \\
\hline$(2,1)$ & {$\left[2 \omega_{2}\right]+1+2 \lambda+\left(\left[2 \omega_{1}\right]+2\right) \mu$} & impossible & \\
\hline$(d, 1)$ & {$\left[d \omega_{2}\right]+1+d \lambda+2 \mu$} & impossible & \\
\hline$(d+1,1)$ & {$\left[(d+1) \omega_{2}\right]+1+(d+1) \lambda+3 \mu$} & impossible & \\
\hline$(2 d+1,2)$ & {$\left[(2 d+1) \omega_{2}\right]+1+(2 d+1) \lambda+\left(\left[(2 d+1) \omega_{1}\right]+2\right) \mu$} & impossible & \\
\hline$(d-1,1)$ & {$\left[(d-1) \omega_{2}\right]+1+(d-1) \lambda+2 \mu$} & impossible & \\
\hline$(3 d+2,3)$ & {$\left[(3 d+2) \omega_{2}\right]+1+(3 d+2) \lambda+\left(\left[(3 d+2) \omega_{1}\right]+2\right) \mu$} & impossible & \\
\hline
\end{tabular}


Table 10 (continued)

\begin{tabular}{|l|l|l|l|}
\hline \multicolumn{1}{|c|}{$(y, z)$} & \multicolumn{1}{|c|}{$\psi_{12, y}=\left[\omega_{2} y\right]+2+y \lambda+\left(\left[\omega_{1} y\right]+1\right) \mu$} & \multicolumn{1}{c|}{$\begin{array}{c}\omega_{2} \lessgtr 1 \\
\text { (1,0) }\end{array}$} & No. \\
\hline \hline$(1,1)$ & {$\left[\omega_{2}\right]+2+\lambda+\mu$} & $>\phi_{6}$ & \\
\hline$(1,-1)$ & {$\left[\omega_{2}\right]+2+\lambda+\mu$} & impossible & \\
\hline$(2,1)$ & {$\left[2 \omega_{2}\right]+2+\lambda+\mu$} & $>\phi_{6}$ & \\
\hline$(d, 1)$ & {$\left[d \omega_{2}\right]+2+d \lambda+\mu$} & $>\phi_{6}$ & \\
\hline$(d+1,1)$ & {$\left[(d+1) \omega_{2}\right]+2+(d+1) \lambda+2 \mu$} & impossible & \\
\hline$(2 d+1,2)$ & {$\left[(2 d+1) \omega_{2}\right]+2+(2 d+1) \lambda+\left(\left[(2 d+1) \omega_{1}\right]+1\right) \mu$} & $>\phi_{6}$ & \\
\hline$(d-1,1)$ & {$\left[(d-1) \omega_{2}\right]+2+(d-1) \lambda+\mu$} & $>\phi_{6}(d \geq 2)$ & \\
\hline$(3 d+2,3)$ & {$\left[(3 d+2) \omega_{2}\right]+2+(3 d+2) \lambda+\left(\left[(3 d+2) \omega_{1}\right]+1\right) \mu$} & $>\phi_{6}$ & \\
\hline
\end{tabular}

\section{Main Theorems}

THEOREM 6.1A. Let $\mathscr{R}=\langle 1, \lambda, \mu\rangle$ be a reduced lattice of $K$ such that $0<\lambda<1,0<X_{\mu}<X_{\lambda}, 0<\omega_{1}(\lambda, \mu)<1, \omega_{2}(\lambda, \mu)>0, a>1,2|b|<1,0<\mu<$ $1, \phi_{1}>1$, where $a=F(\mu), b=Y_{\mu}$. Then

(1) If $F\left(\phi_{1}\right)<1$ :

(i) if $b<0$, then the minimal point adjacent to 1 is $\phi_{1}, \phi_{3}$ or $\phi_{4}$;

(ii) if $b>0$, then the minimal point adjacent to 1 is $\phi_{1}$ or $\phi_{5}$.

(2) If $F\left(\phi_{1}\right)>1, F\left(\phi_{2}\right)<1$ :

(i) if $b<0$, then the minimal point adjacent to 1 is $\phi_{2}$;

(ii) if $b>0$, then the minimal point adjacent to 1 is $\phi_{2}$ or $\phi_{5}$.

(3) If $F\left(\phi_{1}\right)>1, F\left(\phi_{2}\right)>1, F\left(\phi_{6}\right)<1$,

then the minimal point adjacent to 1 is $\phi_{6}$.

Proof. Since $\phi_{1}=\left[\omega_{2}\right]+\lambda>1$, we have $\left[\omega_{2}\right] \geq 1$.

(1) was proved in [5], Theorem 2.1.

(2) We assume that $F\left(\psi_{4,1}\right)>1, F\left(\psi_{5,1}\right)<1$.

(i) the case $b<0$, by Lemma 4.5,(4), we have $\phi_{3}=\psi_{3,1} \neq \theta_{g}$. By Lemma 4.5,(8), we have $\phi_{4}=\psi_{1,1} \neq \theta_{g}$. The others were proved in [5], Theorem 2.1;

(ii) The case $b>0$. The case were all proved in [5], Theorem 2.1.

(3) We assume that $F\left(\psi_{4,1}\right)>1, F\left(\psi_{5,1}\right)>1, F\left(\psi_{8,1}\right)<1$. 
By Lemma 4.5,(1)(2) and Remark 4.4,(1), we have $\theta_{g} \in\left\{\psi_{1, y}, \psi_{2, y}, \psi_{3, y}, \psi_{4, y}\right.$, $\left.\psi_{5, y}, \psi_{6, y}, \psi_{7, y}, \psi_{8,1}\right\}$.

(i) The case $b<0$. By Lemma 4.5,(3), we have $\theta_{g} \in\left\{\psi_{1, y}, \psi_{3, y}, \psi_{4, y}, \psi_{5, y}\right.$, $\left.\psi_{8,1}\right\}$.

Also by Lemma $4.5,(10)$ we have $c_{2}=\left[\omega_{2}\right]-\omega_{2}<-1 / 2$.

(a) In the case of $\psi_{1, y}$, based on Table 1 ,

(1-1) from $\psi_{1,1}=\psi_{8,1}-2$ and $F\left(\psi_{8,1}\right)<1$, we have $F\left(\psi_{1,1}\right)>1$.

(1-2) by Lemma 4.5,(12), $\psi_{1,2}=\left[2 \omega_{2}\right]-1+2 \lambda+\mu=2\left[\omega_{2}\right]+2 \lambda+\mu$ or $2\left[\omega_{2}\right]-1+2 \lambda+\mu$. Since $c_{2}<-1 / 2, \quad \psi_{1,2} \neq 2\left[\omega_{2}\right]-1+2 \lambda+\mu$. Hence $\psi_{1,2}=$ $2\left[\omega_{2}\right]+2 \lambda+\mu>\psi_{8,1}$.

(1-3) $d \geq 2 \Rightarrow \psi_{1, d+1}>\psi_{8,1}$. If $d=1$, then $\psi_{1, d+1}=\psi_{1,2}=\left[2 \omega_{2}\right]-1+2 \lambda+\mu$. This case is just the same as (1-2).

(b) In the case of $\psi_{3, y}$, based on Table 3,

(3-1) by Lemma 4.5,(4) $\phi_{3}=\psi_{3,1} \neq \theta_{g}$.

(c) In the case of $\psi_{4, y}$, based on Table 4 ,

(4-1) by the assumption $\psi_{4,1} \neq \theta_{g}$.

(d) In the case of $\psi_{5, y}$, based on Table 5,

(5-1) by the assumption $\psi_{5,1} \neq \theta_{g}$.

As a result, $\psi_{8,1}$ remains.

(ii) The case $b>0$. By Lemma 4.5,(3), we have $\theta_{g} \in\left\{\psi_{2, y}, \psi_{4, y}, \psi_{5, y}, \psi_{6, y}\right.$, $\left.\psi_{7, y}, \psi_{8,1}\right\}$.

(a) In the case of $\psi_{2, y}$, based on Table 2,

(2-1) by Lemma $4.5,(9), \psi_{2,1} \neq \theta_{g}$.

$(2-2)$ by Lemma $4.5,(12), \psi_{2,2}=\left[2 \omega_{2}\right]-1+2 \lambda+\mu=2\left[\omega_{2}\right]+2 \lambda+\mu\left(>\psi_{8,1}\right)$ or $2\left[\omega_{2}\right]-1+2 \lambda+\mu$.

The case $\psi_{2,2}=2\left[\omega_{2}\right]-1+2 \lambda+\mu$. If $\left[\omega_{2}\right] \geq 2$, then we have $2\left[\omega_{2}\right]-1+$ $2 \lambda+\mu>\psi_{8,1}$. If $\left[\omega_{2}\right]=1$, then $\psi_{2,2}=1+2 \lambda+\mu$. We shall show that $F(1+2 \lambda+\mu)>1$. Since $F\left(\phi_{6}\right)=F(2+\lambda)<1$, we have $-1<Y_{2+\lambda}<1$, so $-3<Y_{\lambda}<-1$. Suppose that $Y_{\lambda}>-3 / 2$. Then $Y_{2+\lambda}=2+Y_{\lambda}>1 / 2$. From this, we have $1 / 4+Z_{2+\lambda}^{2}<Y_{2+\lambda}^{2}+Z_{2+\lambda}^{2}<1$. Hence, $\left|Z_{2+\lambda}\right|<\sqrt{3} / 2$. Since $Y_{\lambda}>-3 / 2$ and $Y_{\lambda}<-1$, we have $-1 / 2<Y_{1+\lambda}<0$. Hence, $F(1+\lambda)=Y_{1+\lambda}^{2}+Z_{1+\lambda}^{2}=$ $Y_{1+\lambda}^{2}+Z_{2+\lambda}^{2}<1 / 4+3 / 4=1$. Since $F\left(\phi_{1}\right)=F(1+\lambda)>1$, we have reached a contradiction. Therefore, we have $Y_{\lambda}<-3 / 2$. From this, we have $Y_{1+2 \lambda+\mu}=$ $1+2 Y_{\lambda}+Y_{\mu}<1-3+Y_{\mu}<-3 / 2$. Hence, $F(1+2 \lambda+\mu)>1$.

(2-3) $d \geq 3 \Rightarrow \psi_{2, d}=\left[d \omega_{2}\right]-1+d \lambda+\mu>\psi_{8,1}$.

The case $d=1,2$ are just the same as (2-1) or (2-2).

(2-5) Similar to (2-3).

(b) In the case of $\psi_{4, y}$, based on Table 4 , 
(4-1) by the assumption, $\psi_{4,1} \neq \theta_{g}$.

(c) In the case of $\psi_{5, y}$, based on Table 5,

(5-1) by the assumption $\psi_{5,1} \neq \theta_{g}$.

(d) In the case of $\psi_{6, y}$, based on Table 6, no case is included

(e) In the case of $\psi_{7, y}$, based on Table 7,

(7-1) by Lemma 4.5,(5), $\psi_{7,1} \neq \theta_{g}$.

As a result, $\psi_{8,1}$ remains.

REMARK. From the proof in [5, Theorem 2.1], (1) and (2) don't require the assumption $0<X_{\mu}<X_{\lambda}$. Moreover, in (1) and (2) (except for the part of $\left.\phi_{4}\right)$, we can weaken the condition from $a>1,2|b|<1$ to $a>$ $\max \left(1,2 b^{2}, 2|b|\right)$.

THEOREM 6.2A. Let $\mathscr{R}=\langle 1, \lambda, \mu\rangle$ be a reduced lattice of $K$ such that $0<\lambda<1,0<X_{\mu}<X_{\lambda}, 0<\omega_{1}(\lambda, \mu)<1, \omega_{2}(\lambda, \mu)>0, a>1,2|b|<1, \mu>1$, $\phi_{1}>1$, where $a=F(\mu), b=Y_{\mu}$. Then

(1) If $F\left(\phi_{1}\right)<1$ :

(i) if $b<0$, then the minimal point adjacent to 1 is $\phi_{1}, \phi_{3}$ or $\phi_{4}$;

(ii) if $b>0$, then the minimal point adjacent to 1 is $\phi_{1}$ or $\phi_{7}$.

(2) If $F\left(\phi_{1}\right)>1, F\left(\phi_{6}\right)<1$ :

(i) if $b<0$, then the minimal point adjacent to 1 is $\phi_{6}$;

(ii) if $b>0$, then the minimal point adjacent to 1 is $\phi_{5}$ or $\phi_{6}$.

Proof. Since $\phi_{1}=\psi_{4,1}=\left[\omega_{2}\right]+\lambda>1$, we have $\left[\omega_{2}\right] \geq 1$.

(1) We assume that $F\left(\psi_{4,1}\right)<1$.

By Lemma 4.5,(1)(2) and Remark 4.4,(2), we have $\theta_{g} \in\left\{\psi_{1, y}, \psi_{3, y}, \psi_{7, y}, \psi_{4,1}\right\}$.

(i) The case $b<0$. By Lemma 4.5,(3), we have $\theta_{g} \in\left\{\psi_{1, y}, \psi_{3, y}, \psi_{4,1}\right\}$.

(a) In the case of $\psi_{1, y}$, based on Table 1 ,

(1-1) $\psi_{1,1}$.

$(1-2) \psi_{1,2}=\left[2 \omega_{2}\right]-1+2 \lambda+\mu>\psi_{8,1}$.

(1-3) $\psi_{1, d+1}>\psi_{8,1}>\psi_{4,1}$.

(b) In the case of $\psi_{3, y}$, based on Table 3,

(3-1) $\psi_{3,1}$.

As a result, $\psi_{4,1}, \psi_{3,1}$ and $\psi_{1,1}$ remain.

(ii) The case $b>0$. By Lemma 4.5,(3), we have $\theta_{g} \in\left\{\psi_{7, y}, \psi_{4,1}\right\}$.

(a) In the case of $\psi_{7, y}$, based on Table 7,

(7-1) $\psi_{7,1}$. 
As a result, $\psi_{4,1}$ and $\psi_{7,1}$ remain.

(2) We assume that $F\left(\psi_{4,1}\right)>1, F\left(\psi_{8,1}\right)<1$.

By Lemma 4.5,(1)(2) and Remark 4.4,(2), we have $\theta_{g} \in\left\{\psi_{1, y}, \psi_{2, y}, \psi_{3, y}, \psi_{4, y}\right.$, $\left.\psi_{7, y}, \psi_{8,1}\right\}$.

(i) The case $b<0$. By Lemma 4.5,(3), we have $\theta_{g} \in\left\{\psi_{1, y}, \psi_{3, y}, \psi_{4, y}, \psi_{8,1}\right\}$.

(a) In the case of $\psi_{1, y}$, based on Table 1,

(1-1) from $\psi_{1,1}=\psi_{8,1}-2$ and $F\left(\psi_{8,1}\right)<1$, we have $F\left(\psi_{1,1}\right)>1$.

$(1-2) \psi_{1,2}=\left[2 \omega_{2}\right]-1+2 \lambda+\mu>\psi_{8,1}$.

(1-3) $\psi_{1, d+1}>\psi_{8,1}$.

(b) In the case of $\psi_{3, y}$, based on Table 3,

(3-1) by Lemma 4.5,(4) $\phi_{3}=\psi_{3,1} \neq \theta_{g}$.

(c) In the case of $\psi_{4, y}$, based on Table 4 ,

(4-1) by the assumption $\psi_{4,1} \neq \theta_{g}$.

As a result, $\psi_{8,1}$ remains.

(ii) The case $b>0$. By Lemma 4.5,(3), we have $\theta_{g} \in\left\{\psi_{2, y}, \psi_{4, y}, \psi_{7, y}, \psi_{8,1}\right\}$.

(a) In the case of $\psi_{2, y}$, based on Table 2,

(2-1) $\psi_{2,1}=\left[\omega_{2}\right]-1+\lambda+\mu\left(>\psi_{4,1}\right)$.

(2-2) $\psi_{2,2}=\left[2 \omega_{2}\right]-1+2 \lambda+\mu>\psi_{8,1}$.

(2-3) $d \geq 3 \Rightarrow \psi_{2, d}=\left[d \omega_{2}\right]-1+d \lambda+\mu>\psi_{8,1}$.

The cases $d=1,2$ are just the same as (2-1) or (2-2).

(2-5) Similar to (2-3).

(b) In the case of $\psi_{4, y}$, based on Table 4,

(4-1) by the assumption $\psi_{4,1} \neq \theta_{g}$.

(c) In the case of $\psi_{7, y}$, based on Table 7,

(7-1) by Lemma $4.5,(5) \psi_{7,1} \neq \theta_{g}$.

As a result, $\psi_{8,1}$ and $\psi_{2,1}$ remain.

THEOREM 6.3A. Let $\mathscr{R}=\langle 1, \lambda, \mu\rangle$ be a reduced lattice of $K$ such that $0<\lambda<1,0<X_{\mu}<X_{\lambda}, 0<\omega_{1}(\lambda, \mu)<1, \omega_{2}(\lambda, \mu)>0, a>1,2|b|<1, \mu<0$, $\phi_{1}>1$, where $a=F(\mu), b=Y_{\mu}$. Then

(1) If $F\left(\phi_{1}\right)<1$ :

(i) if $\left[\omega_{2}\right] \geq 2$, then the minimal point adjacent to 1 is $\phi_{1}, \phi_{2}$ or $\phi_{4}$;

(ii-a) if $\left[\omega_{2}\right]=1, \lambda+\mu<0$, then the minimal point adjacent to 1 is $\phi_{1}$ or $1+\phi_{9}$

(ii-b) if $\left[\omega_{2}\right]=1, \lambda+\mu>0$, then the minimal point adjacent to 1 is $\phi_{1}$ or $\phi_{2}$.

(2) If $F\left(\phi_{1}\right)>1, F\left(\phi_{6}\right)<1$, then the minimal point adjacent to 1 is $\phi_{2}, \phi_{6}$ or $\phi_{8}$. 
Proof. Since $\mu<0$ and $0<X_{\mu}$, we have $b<0$ and $-1 / 2<\mu$.

From Table 10 and Lemma 4.5,(3), we have $\theta_{g} \in\left\{\psi_{1, y}, \psi_{3, y}, \psi_{4, y}, \psi_{5, y}\right.$, $\left.\psi_{8, y}, \psi_{9, y}\right\}$.

(1) We assume that $F\left(\psi_{4,1}\right)<1$.

(a) In the case of $\psi_{1, y}$, based on Table 1 ,

(1-1) $\psi_{1,1}$.

(1-2) by Lemma $4.5,(12) \quad \psi_{1,2}=\left[2 \omega_{2}\right]-1+2 \lambda+\mu=2\left[\omega_{2}\right]+2 \lambda+\mu\left(>\psi_{4,1}\right)$ or $2\left[\omega_{2}\right]-1+2 \lambda+\mu$.

The case $\psi_{1,2}=2\left[\omega_{2}\right]-1+2 \lambda+\mu$. If $\left[\omega_{2}\right] \geq 2$, then we have $\psi_{1,2}>\psi_{4,1}$. If $\left[\omega_{2}\right]=1, \psi_{1,2}=1+2 \lambda+\mu$.

$(1-3) d \geq 2 \Rightarrow \psi_{1, d+1} \geq\left[3 \omega_{2}\right]-1+3 \lambda+\mu>\psi_{4,1}$. The case $d=1$ is just the same as $(1-2)$.

(1-4) $\psi_{1,2 d+1}>\psi_{4,1}$.

(b) In the case of $\psi_{3, y}$, based on Table 3,

(3-1) $\psi_{3,1}=\left[\omega_{2}\right]+\lambda-\mu>\left[\omega_{2}\right]+\lambda=\psi_{4,1}$.

(c) In the case of $\psi_{4, y}$, based on Table 4,

(4-1) $\psi_{4,1}$.

(4-2) $\psi_{4,2}=\left[2 \omega_{2}\right]+2 \lambda+\mu>\psi_{4,1}$.

(4-3) $\psi_{4, d+1}>\psi_{4,1}$.

(d) In the case of $\psi_{5, y}$, based on Table 5,

(5-1) $\psi_{5,1}=\left[\omega_{2}\right]+\lambda+\mu$.

(5-2) $\psi_{5,2}=\left[2 \omega_{2}\right]+2 \lambda+\mu>\psi_{4,1}$.

$(5-3) d \geq 2 \Rightarrow \psi_{5, d} \geq\left[2 \omega_{2}\right]+2 \lambda+\mu>\psi_{4,1}$.

The case $d=1$ is just the same as (5-1).

(5-5) Similar to (5-3).

(e) In the case of $\psi_{8, y}$, based on Table 8 ,

(8-1) $\psi_{8,1}>\psi_{4,1}$.

(f) In the case of $\psi_{9, y}$, based on Table 9,

(9-1) $\psi_{9,1}=\left[\omega_{2}\right]+1+\lambda+\mu>\psi_{4,1}$.

As a result, $\psi_{4,1}, \psi_{5,1}, \psi_{1,1}$ and $1+2 \lambda+\mu$ remain. Moreover, If $\left[\omega_{2}\right] \geq 2$, then we have $\theta_{g} \neq 1+2 \lambda+\mu$. The case $\left[\omega_{2}\right]=1$. Since $\phi_{4}=\psi_{1,1}=\left[\omega_{2}\right]-1+\lambda$ $=\lambda<1$, we have $\theta_{g} \neq \psi_{1,1}$. If $\lambda+\mu<0$, then we have $\phi_{2}=1+\lambda+\mu<1$. If $\lambda+\mu>0$, then we have $1+2 \lambda+\mu \neq \theta_{g}$, because $1+2 \lambda+\mu=1+\lambda+(\lambda+\mu)>$ $1+\lambda=\psi_{4,1}$.

(2) We assume that $F\left(\phi_{1}\right)>1, F\left(\phi_{6}\right)<1$.

We note that by Lemma $4.5,(10)$, we have $c_{2}=\left[\omega_{2}\right]-\omega_{2}<-1 / 2$. So by Lemma $4.5,(12)$, we have $\left[2 \omega_{2}\right]=2\left[\omega_{2}\right]+1$.

(a) In the case of $\psi_{1, y}$, based on Table 1 , 
(1-1) from $\psi_{1,1}=\psi_{8,1}-2$ and $F\left(\psi_{8,1}\right)<1$, we have $F\left(\psi_{1,1}\right)>1$.

(1-2) $\psi_{1,2}=\left[2 \omega_{2}\right]-1+2 \lambda+\mu=2\left[\omega_{2}\right]+2 \lambda+\mu$. If such a $\psi_{1,2}$ exist, then by $\left[2 \omega_{1}\right]=1$, we have $c_{1}<-1 / 2\left(\Leftrightarrow\left[2 \omega_{1}\right]=1\right)$.

(i) The case $\left[\omega_{2}\right] \geq 2$. We have $\psi_{1,2}>\psi_{8,1}$.

(ii) The case $\left[\omega_{2}\right]=1 . \psi_{1,2}=2+2 \lambda+\mu>2+\lambda+\mu=\psi_{9,1}$.

From Lemma 4.5,(11), we have $F\left(\psi_{9,1}\right)<F\left(\psi_{8,1}\right)$. So we have $F\left(\psi_{9,1}\right)<1$. Therefore, $\psi_{1,2}=2+2 \lambda+\mu \neq \theta_{g}$.

(1-3) (i) The case $d \geq 2$. We have $\psi_{1, d+1} \geq\left[3 \omega_{2}\right]-1+3 \lambda+\mu \geq\left[2 \omega_{2}\right]+$ $\left[\omega_{2}\right]-1+3 \lambda+\mu=3\left[\omega_{2}\right]+3 \lambda+\mu>\psi_{8,1}$.

(ii) The case $d=1$. Since $d=1 \Leftrightarrow\left[2 \omega_{1}\right]=1$, this case is just the same as (1-2).

$(1-4) \psi_{1,2 d+1} \geq\left[3 \omega_{2}\right]-1+3 \lambda+2 \mu \geq\left[2 \omega_{2}\right]+\left[\omega_{2}\right]-1+3 \lambda+2 \mu=3\left[\omega_{2}\right]+3 \lambda$ $+2 \mu>\psi_{8,1}$.

(b) In the case of $\psi_{3, y}$, based on Table 3,

(3-1) by Lemma 4.5,(4) $\phi_{3}=\psi_{3,1} \neq \theta_{g}$.

(c) In the case of $\psi_{4, y}$, based on Table 4 ,

(4-1) $F\left(\psi_{4,1}\right)>1$.

(4-2) $\psi_{4,2}=\left[2 \omega_{2}\right]+2 \lambda+\mu=2\left[\omega_{2}\right]+1+2 \lambda+\mu>\psi_{8,1}$.

(4-3) $\psi_{4, d+1} \geq\left[2 \omega_{2}\right]+2 \lambda+\mu>\psi_{8,1}$.

(d) In the case of $\psi_{5, y}$, based on Table 5,

(5-1) $\psi_{5,1}=\left[\omega_{2}\right]+\lambda+\mu$.

$(5-2) \psi_{5,2}=\left[2 \omega_{2}\right]+2 \lambda+\mu=2\left[\omega_{2}\right]+1+2 \lambda+\mu>\psi_{8,1}$.

(5-3) $d \geq 2 \Rightarrow \psi_{5, d} \geq\left[2 \omega_{2}\right]+2 \lambda+\mu>\psi_{8,1}$.

The case $d=1$ is just the same as (5-1).

(5-5) Similar to (5-3).

(e) In the case of $\psi_{8, y}$, based on Table 8 ,

(8-1) $F\left(\psi_{8,1}\right)<1$.

(f) In the case of $\psi_{9, y}$, based on Table 9 ,

(9-1) $\psi_{9,1}=\left[\omega_{2}\right]+1+\lambda+\mu$.

As a result, $\psi_{8,1}, \psi_{5,1}$ and $\psi_{9,1}$ remain.

THEOREM 6.1B. Let $\mathscr{R}=\langle 1, \lambda, \mu\rangle$ be a reduced lattice of $K$ such that $0<\lambda<1,0<X_{\mu}<X_{\lambda}, 0<\omega_{1}(\lambda, \mu)<1, \omega_{2}(\lambda, \mu)>0, a>1,2|b|<1,0<\mu<$ $1, \phi_{1}<1, F\left(\phi_{6}\right)<1$, where $a=F(\mu), b=Y_{\mu}$. Then

(1) If $F\left(\phi_{2}\right)<1$, then the minimal point adjacent to 1 is $\phi_{2}$.

(2) If $\phi_{2}>1, F\left(\phi_{2}\right)>1$, then the minimal point adjacent to 1 is $\phi_{6}$.

(3) If $\phi_{2}<1$ :

(i) if $b<0$, then the minimal point adjacent to 1 is $\phi_{6}$; 
(ii-a) if $b>0,2 \lambda+\mu<1$, then the minimal point adjacent to 1 is $\phi_{6}$ or $\phi_{10}$

(ii-b) if $b>0,2 \lambda+\mu>1$, then the minimal point adjacent to 1 is $\phi_{6}$ or $\phi_{9}$.

Proof. From the assumption $\phi_{1}<1$, by Lemma 5.2,(1), we have $Y_{\lambda}<$ $-1 / 2$. By Corollary 5.3, if $b<0$, then we have $1>\omega_{2}>1 / 2$.

(1) We assume that $F\left(\psi_{5,1}\right)<1$. Since $\mathscr{R}$ is a reduced lattice, we have $\psi_{5,1}=\left[\omega_{2}\right]+\lambda+\left(\left[\omega_{1}\right]+1\right) \mu=\lambda+\mu>1$.

By Lemma 4.5,(1)(2) and Remark 4.4,(1) we have $\theta_{g} \in\left\{\psi_{1, y}, \psi_{2, y}, \psi_{3, y}, \psi_{4, y}\right.$, $\left.\psi_{7, y}, \psi_{5,1}\right\}$.

(i) The case $b<0$. By Lemma 4.5,(3), we have $\theta_{g} \in\left\{\psi_{1, y}, \psi_{3, y}, \psi_{4, y}, \psi_{5,1}\right\}$.

(a) In the case of $\psi_{1, y}$, based on Table 1 ,

$(1-2)$ since $\left[2 \omega_{2}\right]=1$, we have $\psi_{1,2}=2 \lambda+\mu>\psi_{8,1}>\psi_{5,1}$.

$(1-3)\left[(d+1) \omega_{2}\right] \geq 2 \Rightarrow \psi_{1, d+1}>\psi_{8,1}>\psi_{5,1} . \quad\left[(d+1) \omega_{2}\right]=1 \Rightarrow \psi_{1, d+1}=$ $(d+1) \lambda+\mu \Rightarrow Y_{\psi_{1, d+1}}=(d+1) Y_{\lambda}+Y_{\mu}<-1$.

$(1-4)\left[(2 d+1) \omega_{2}\right] \geq 2 \Rightarrow \psi_{1,2 d+1}>\psi_{8,1}>\psi_{5,1} \cdot\left[(2 d+1) \omega_{2}\right]=1 \Rightarrow \psi_{1,2 d+1}=$ $(2 d+1) \lambda+2 \mu>\psi_{8,1}>\psi_{5,1}$.

(1-5) from $\left[(3 d+2) \omega_{2}\right] \geq 2$, we have $\psi_{1,3 d+2} \geq 1+(3 d+2) \lambda+3 \mu>\psi_{8,1}>$ $\psi_{5,1}$.

(b) In the case of $\psi_{3, y}$, based on Table 3,

(3-2) $\psi_{3,3 d+2}>\psi_{8,1}>\psi_{5,1}$.

(c) In the case of $\psi_{4, y}$, based on Table 4 ,

(4-2) since $\left[2 \omega_{2}\right]=1$, we have $\psi_{4,2}=1+2 \lambda+\mu>\psi_{8,1}>\psi_{5,1}$.

(4-3) $\psi_{4, d+1}>\psi_{8,1}>\psi_{5,1}$.

(4-4) $\psi_{4,2 d+1}>\psi_{8,1}>\psi_{5,1}$.

(4-5) $\psi_{4,3 d+2}>\psi_{8,1}>\psi_{5,1}$.

(ii) The case $b>0$. By Lemma 4.5,(3), we have $\theta_{g} \in\left\{\psi_{2, y}, \psi_{4, y}, \psi_{7, y}, \psi_{5,1}\right\}$.

(a) In the case of $\psi_{2, y}$, based on Table 2,

(2-1) $\psi_{2,1}=-1+\lambda+\mu<1$.

(2-2) $\left[2 \omega_{2}\right]=0 \Rightarrow \psi_{2,2}=-1+2 \lambda+\mu \Rightarrow Y_{\psi_{2,2}}=-1+2 Y_{\lambda}+Y_{\mu}<-1 .\left[2 \omega_{2}\right]=$ $1 \Rightarrow \psi_{2,2}=2 \lambda+\mu>\psi_{8,1}>\psi_{5,1}$.

$(2-3)\left[d \omega_{2}\right] \geq 2 \Rightarrow \psi_{2, d}>\psi_{8,1}>\psi_{5,1} . \quad\left[d \omega_{2}\right]=1 \Rightarrow$ Since $\quad d \geq 2, \quad \psi_{2, d}=$ $d \lambda+\mu>\psi_{8,1}>\psi_{5,1} . \quad\left[d \omega_{2}\right]=0 \Rightarrow \psi_{2, d}=-1+d \lambda+\mu \Rightarrow Y_{\psi_{2, d}}=-1+d Y_{\lambda}+Y_{\mu}$ $<-1$.

$(2-4)\left[(2 d+1) \omega_{2}\right] \geq 2 \Rightarrow \psi_{2,2 d+1}>\psi_{8,1}>\psi_{5,1} \cdot\left[(2 d+1) \omega_{2}\right]=1 \Rightarrow \psi_{2,2 d+1}=$ $(2 d+1) \lambda+2 \mu>\psi_{8,1}>\psi_{5,1} . \quad\left[(2 d+1) \omega_{2}\right]=0 \Rightarrow \psi_{2,2 d+1}=-1+(2 d+1) \lambda+2 \mu$ $\Rightarrow Y_{\psi_{2,2 d+1}}=-1+(2 d+1) Y_{\lambda}+2 Y_{\mu}<-1$. 
(2-5) Similar to (2-3).

$(2-6)\left[(3 d+2) \omega_{2}\right] \geq 2 \Rightarrow \psi_{2,3 d+2}>\psi_{8,1}>\psi_{5,1}$. $\left[(3 d+2) \omega_{2}\right]=1 \Rightarrow \psi_{2,3 d+2}=$ $(3 d+2) \lambda+3 \mu>\psi_{8,1}>\psi_{5,1} . \quad\left[(3 d+2) \omega_{2}\right]=0 \Rightarrow \psi_{2,3 d+2}=-1+(3 d+2) \lambda+3 \mu$ $\Rightarrow Y_{\psi_{2,3 d+2}}=-1+(3 d+2) Y_{\lambda}+3 Y_{\mu}<-1$.

(b) In the case of $\psi_{4, y}$, based on Table 4 ,

(4-2) $\left[2 \omega_{2}\right]=0 \Rightarrow \psi_{4,2}=2 \lambda+\mu>\psi_{8,1}>\psi_{5,1}$. $\left[2 \omega_{2}\right]=1 \Rightarrow \psi_{4,2}=1+2 \lambda+\mu$ $>\psi_{8,1}>\psi_{5,1}$.

(4-3) $\psi_{4, d+1}>\psi_{8,1}>\psi_{5,1}$.

(4-4) $\psi_{4,2 d+1}>\psi_{8,1}>\psi_{5,1}$.

(4-5) $\psi_{4,3 d+2}>\psi_{8,1}>\psi_{5,1}$.

(c) In the case of $\psi_{7, y}$, based on Table 7,

(7-1) by Lemma $4.5,(5) \psi_{7,1} \neq \theta_{g}$.

As a result, $\psi_{5,1}$ remains.

(2) We assume that $\psi_{5,1}=\lambda+\mu>1, F\left(\psi_{5,1}\right)>1$.

By Lemma 4.5,(1)(2) and Remark 4.4,(1) we have $\theta_{g} \in\left\{\psi_{1, y}, \psi_{2, y}, \psi_{3, y}, \psi_{4, y}\right.$, $\left.\psi_{5, y}, \psi_{6, y}, \psi_{7, y}, \psi_{8,1}\right\}$.

(i) The case $b<0$. By Lemma 4.5,(3), we have $\theta_{g} \in\left\{\psi_{1, y}, \psi_{3, y}, \psi_{4, y}, \psi_{5, y}\right.$, $\left.\psi_{8,1}\right\}$.

(a) In the case of $\psi_{1, y}$, based on Table 1, similar to (1).

(b) In the case of $\psi_{3, y}$, based on Table 3, similar to (1).

(c) In the case of $\psi_{4, y}$, based on Table 4 , similar to (1).

(d) In the case of $\psi_{5, y}$, based on Table 5,

(5-1) from the assumption, $F\left(\psi_{5,1}\right)>1$.

(5-2) $\psi_{5,2}>\phi_{6}$. (5-3) $\psi_{5, d}>\phi_{6}(d \geq 2)$.

(5-4) $\psi_{5,2 d+1}>\phi_{6} .(5-5) \psi_{5, d-1}>\phi_{6}(d \geq 3)$.

As a result, $\psi_{8,1}$ remains.

(ii) The case $b>0$. By Lemma 4.5,(3), we have $\theta_{g} \in\left\{\psi_{2, y}, \psi_{4, y}, \psi_{5, y}, \psi_{6, y}\right.$, $\left.\psi_{7, y}, \psi_{8,1}\right\}$.

(a) In the case of $\psi_{2, y}$, based on Table 2, similar to (1).

(b) In the case of $\psi_{4, y}$, based on Table 4 , similar to (1).

(c) In the case of $\psi_{5, y}$, based on Table 5,

(5-1) from the assumption, $F\left(\psi_{5,1}\right)>1$.

(5-2) $\psi_{5,2}>\phi_{6}$. (5-3) $\psi_{5, d}>\phi_{6}(d \geq 2)$. 
(5-4) $\psi_{5,2 d+1}>\phi_{6}$. (5-5) $\psi_{5, d-1}>\phi_{6}(d \geq 3)$.

(d) In the case of $\psi_{6, y}$, based on Table 6, no case included

(e) In the case of $\psi_{7, y}$, based on Table 7, similar to (1).

As a result, $\psi_{8,1}$ remains.

(3) We assume that $\psi_{5,1}<1$.

By Lemma 4.5,(1)(2) and Remark 4.4,(1) we have $\theta_{g} \in\left\{\psi_{1, y}, \psi_{2, y}, \psi_{3, y}, \psi_{4, y}\right.$, $\left.\psi_{5, y}, \psi_{6, y}, \psi_{7, y}, \psi_{8,1}\right\}$.

(i) The case $b<0$. By Lemma 4.5,(3), we have $\theta_{g} \in\left\{\psi_{1, y}, \psi_{3, y}, \psi_{4, y}, \psi_{5, y}\right.$, $\left.\psi_{8,1}\right\}$.

(a) In the case of $\psi_{1, y}$, based on Table 1,

(1-2) $\psi_{1,2}=2 \lambda+\mu, Y_{\psi_{1,2}}=2 Y_{\lambda}+Y_{\mu}<-1$.

(1-3) The case $d \geq 3$. $\psi_{1, d+1}>1+4 \lambda+\mu>\phi_{6}$. The case $d=2 . \psi_{1, d+1}=$ $\left[3 \omega_{2}\right]-1+3 \lambda+\mu . \quad\left[3 \omega_{2}\right]=2 \Rightarrow \psi_{1, d+1}=1+3 \lambda+\mu>\phi_{6} . \quad\left[3 \omega_{2}\right]=1 \Rightarrow \psi_{1, d+1}=$ $3 \lambda+\mu . \quad Y_{\psi_{1, d+1}}=3 Y_{\lambda}+Y_{\mu}<-1$.

(1-4) The case $d \geq 2 . \psi_{1,2 d+1}>\phi_{6}$.

The case $d=1 . \quad \psi_{1,2 d+1}=\left[3 \omega_{2}\right]-1+3 \lambda+2 \mu . \quad\left[3 \omega_{2}\right]=2 \Rightarrow \psi_{1,2 d+1}=1+$ $3 \lambda+2 \mu>\phi_{6} .\left[3 \omega_{2}\right]=1 \Rightarrow \psi_{1,2 d+1}=3 \lambda+2 \mu . \quad Y_{\psi_{1,2 d+1}}=3 Y_{\lambda}+2 Y_{\mu}<-1$.

(1-5) $\psi_{1,3 d+2}>\phi_{6}$.

(b) In the case of $\psi_{3, y}$, based on Table 3,

(3-2) $\psi_{3,3 d+2}>\phi_{6}$.

(c) In the case of $\psi_{4, y}$, based on Table 4,

(4-2) $\psi_{4,2}>\phi_{6}$. (4-3) $\psi_{4, d+1}>\phi_{6}$. (4-4) $\psi_{4,2 d+1}>\phi_{6}$. (4-5) $\psi_{4,3 d+2}>\phi_{6}$.

(d) In the case of $\psi_{5, y}$, based on Table 5,

(5-1) from the assumption, $\psi_{5,1}<1$. (5-2) $\psi_{5,2}>\phi_{6}$.

(5-3) $\psi_{5, d}>\phi_{6}(d \geq 2)$. (5-4) $\psi_{5,2 d+1}>\phi_{6}$. (5-5) $\psi_{5, d-1}>\phi_{6}(d \geq 3)$.

As a result, $\psi_{8,1}$ remains.

(ii) The case $b>0$. by Lemma $4.5,(3)$, we have $\theta_{g} \in\left\{\psi_{2, y}, \psi_{4, y}, \psi_{5, y}, \psi_{6, y}\right.$, $\left.\psi_{7, y}, \psi_{8,1}\right\}$.

(a) In the case of $\psi_{2, y}$, based on Table 2,

(2-1) $\psi_{2,1}=-1+\lambda+\mu<1$.

$(2-2)\left[2 \omega_{2}\right]=0 \Rightarrow \psi_{2,2}=-1+2 \lambda+\mu<\lambda<1 .\left[2 \omega_{2}\right]=1 \Rightarrow \psi_{2,2}=2 \lambda+\mu$.

(2-3) The case $\left[d \omega_{2}\right] \geq 2 . \psi_{2, d}>\psi_{8,1}>\psi_{5,1}$.

The case $\left[d \omega_{2}\right]=1$. We have $d \geq 2 \Rightarrow \psi_{2, d}=d \lambda+\mu$. If $d \geq 3$, then we have $Y_{\psi_{2, d}}=d Y_{\lambda}+Y_{\mu}<-1$. Hence, only when $d=2$, it is possible to have $\theta_{g}=\psi_{2, d}=\psi_{2,2}=2 \lambda+\mu$. The case $\left[d \omega_{2}\right]=0 . \quad \psi_{2, d}=-1+d \lambda+\mu . \quad Y_{\psi_{2, d}}=$ $-1+d Y_{\lambda}+Y_{\mu}<-1$. 
(2-4) The case $\left[(2 d+1) \omega_{2}\right] \geq 2 . \psi_{2,2 d+1}>\psi_{8,1}$. The case $\left[(2 d+1) \omega_{2}\right]=1$. $\psi_{2,2 d+1}=(2 d+1) \lambda+2 \mu$. If $d \geq 2$, then we have $Y_{\psi_{2,2 d+1}}=(2 d+1) Y_{\lambda}+2 Y_{\mu}$ $<-1$. Hence, only when $d=1$, it is possible to have $\theta_{g}=\psi_{2,3}=3 \lambda+2 \mu$. The case $\left[(2 d+1) \omega_{2}\right]=0 . \quad \psi_{2,2 d+1}=-1+(2 d+1) \lambda+2 \mu . \quad Y_{\psi_{2,2 d+1}}=-1+$ $(2 d+1) Y_{\lambda}+2 Y_{\mu}<-1$.

(2-5) Similar to (2-3).

$(2-6)$ The case $\left[(3 d+2) \omega_{2}\right] \geq 2 . \quad \psi_{2,3 d+2}>\psi_{8,1}$. The case $\left[(3 d+2) \omega_{2}\right]$ $=1 . \quad \psi_{2,3 d+2}=(3 d+2) \lambda+3 \mu . \quad Y_{\psi_{2,3 d+2}}=(3 d+2) Y_{\lambda}+3 Y_{\mu}<-1 . \quad$ The case $\left[(3 d+2) \omega_{2}\right]=0 . \quad \psi_{2,3 d+2}=-1+(3 d+2) \lambda+3 \mu . \quad Y_{\psi_{2,3 d+2}}=-1+(3 d+2) Y_{\lambda}+$ $3 Y_{\mu}<-1$.

(b) In the case of $\psi_{4, y}$, based on Table 4 ,

(4-2) $\left[2 \omega_{2}\right]=0 \Rightarrow \psi_{4,2}=2 \lambda+\mu$. $\left[2 \omega_{2}\right]=1 \Rightarrow \psi_{4,2}=1+2 \lambda+\mu>\psi_{8,1}$.

(4-3) The case $\left[(d+1) \omega_{2}\right] \geq 1 . \quad \psi_{4, d+1}>\psi_{8,1}$. The case $\left[(d+1) \omega_{2}\right]=0$. $\psi_{4, d+1}=(d+1) \lambda+\mu$. If $d \geq 2$, then we have $Y_{\psi_{4, d+1}}=(d+1) Y_{\lambda}+Y_{\mu}<-1$. Hence, only when $d=1$, it is possible to have $\theta_{g}=\psi_{4,2}=2 \lambda+\mu$.

(4-4) The case $\left[(2 d+1) \omega_{2}\right] \geq 1 . \psi_{4,2 d+1}>\psi_{8,1}$. The case $\left[(2 d+1) \omega_{2}\right]=0$. $\psi_{4,2 d+1}=(2 d+1) \lambda+2 \mu$. If $d \geq 2$, then we have $Y_{\psi_{4,2 d+1}}=(2 d+1) Y_{\lambda}+2 Y_{\mu}<$ -1 . Hence, only when $d=1$, it is possible to have $\theta_{g}=\psi_{4,3}=3 \lambda+2 \mu$.

(4-5) $\left[(3 d+2) \omega_{2}\right] \geq 1 \Rightarrow \psi_{4,3 d+2}>\psi_{8,1}$. [(3d+2) $\left.\omega_{2}\right]=0 \Rightarrow \psi_{4,3 d+2}=(3 d+2) \lambda$ $+3 \mu . \quad Y_{\psi_{4,3 d+2}}=(3 d+2) Y_{\lambda}+3 Y_{\mu}<-1$.

(c) In the case of $\psi_{5, y}$, based on Table 5,

(5-1) from the assumption, $F\left(\psi_{5,1}\right)>1$.

$(5-2)\left[2 \omega_{2}\right]=0 \Rightarrow \psi_{5,2}=2 \lambda+\mu .\left[2 \omega_{2}\right]=1 \Rightarrow \psi_{5,2}=1+2 \lambda+\mu>\psi_{8,1}$.

(5-3) The case $\left[d \omega_{2}\right] \geq 1 . \psi_{5, d}>\psi_{8,1}$.

The case $\left[d \omega_{2}\right]=0 . \quad \psi_{5, d}=d \lambda+\mu$. If $d \geq 3$, then we have $Y_{\psi_{5, d}}=$ $d Y_{\lambda}+Y_{\mu}<-1$. Hence, only when $d=2$, it is possible to have $\theta_{g}=\psi_{5,2}=$ $2 \lambda+\mu$.

(5-4) The case $\left[(2 d+1) \omega_{2}\right] \geq 1 . \psi_{5,2 d+1}>\psi_{8,1}$. The case $\left[(2 d+1) \omega_{2}\right]=0$. $\psi_{5,2 d+1}=(2 d+1) \lambda+2 \mu$. If $d \geq 2$, then we have $Y_{\psi_{5,2 d+1}}=(2 d+1) Y_{\lambda}+2 Y_{\mu}<$ -1 . Hence, only when $d=1$, it is possible to have $\theta_{g}=\psi_{5,3}=3 \lambda+2 \mu$.

(5-5) The case $\left[(d-1) \omega_{2}\right] \geq 1 . \quad \psi_{5, d-1}>\psi_{8,1}$. The case $\left[(d-1) \omega_{2}\right]=0$. $\psi_{5, d-1}=(d-1) \lambda+\mu$. If $d \geq 4$, then we have $Y_{\psi_{5, d-1}}=(d-1) Y_{\lambda}+Y_{\mu}<-1$. Hence, only when $d=3$, it is possible to have $\theta_{g}=\psi_{5,2}=2 \lambda+\mu$.

(d) In the case of $\psi_{6, y}$. based on Table 6, no case included

(e) In the case of $\psi_{7, y}$, based on Table 7,

(7-1) By Lemma 4.5,(5) $\psi_{7,1} \neq \theta_{g}$.

As a result, $2 \lambda+\mu, 3 \lambda+2 \mu$ and $\psi_{8,1}$ remain. If $2 \lambda+\mu<1$, then we have 
$2 \lambda+\mu \neq \theta_{g}$. If $2 \lambda+\mu>1$, then we have $3 \lambda+2 \mu \neq \theta_{g}$, because $3 \lambda+2 \mu=$ $(2 \lambda+\mu)+\lambda+\mu>1+\lambda=\psi_{8,1}$.

THEOREM 6.2B. Let $\mathscr{R}=\langle 1, \lambda, \mu\rangle$ be a reduced lattice of $K$ such that $0<\lambda<1,0<X_{\mu}<X_{\lambda}, 0<\omega_{1}(\lambda, \mu)<1, \omega_{2}(\lambda, \mu)>0, a>1,2|b|<1, \mu>1$, $\phi_{1}<1, F\left(\phi_{6}\right)<1$, where $a=F(\mu), b=Y_{\mu}$. Then the minimal point adjacent to 1 is $\phi_{6}$.

Proof. From the assumption $\phi_{1}<1$, by Lemma 5.2,(1), we have $Y_{\lambda}<$ $-1 / 2$. By Corollary 5.3, if $b<0$, then we have $\omega_{2}>1 / 2$.

By Lemma 4.5,(1)(2) and Remark 4.4,(2) we have $\theta_{g} \in\left\{\psi_{1, y}, \psi_{2, y}, \psi_{3, y}, \psi_{4, y}\right.$, $\left.\psi_{7, y}, \psi_{8,1}\right\}$.

(i) The case $b<0$. By Lemma 4.5,(3), we have $\theta_{g} \in\left\{\psi_{1, y}, \psi_{3, y}, \psi_{4, y}, \psi_{8,1}\right\}$.

(a) In the case of $\psi_{1, y}$, based on Table 1 ,

(1-1) from $\psi_{1,1}=\psi_{8,1}-2$ and $F\left(\psi_{8,1}\right)<1$, we have $F\left(\psi_{1,1}\right)>1$.

(1-2) $\psi_{1,2}=2 \lambda+\mu>\psi_{8,1} \cdot(1-3) \psi_{1, d+1}>\psi_{8,1}$.

(1-4) $\psi_{1,2 d+1}>\psi_{8,1}$. (1-5) $\psi_{1,3 d+2}>\psi_{8,1}$.

(b) In the case of $\psi_{3, y}$, based on Table 3,

(3-2) $\psi_{3,3 d+2}>\psi_{8,1}$.

(c) In the case of $\psi_{4, y}$, based on Table 4 ,

(4-2) $\psi_{4,2}>\psi_{8,1}$. (4-3) $\psi_{4,3}>\psi_{8,1}$.

(4-4) $\psi_{4,2 d+1}>\psi_{8,1}$. (4-5) $\psi_{4,3 d+2}>\psi_{8,1}$.

As a result $\psi_{8,1}$ remains.

(ii) The case $b>0$. By Lemma 4.5,(3), we have $\theta_{g} \in\left\{\psi_{2, y}, \psi_{4, y}, \psi_{7, y}, \psi_{8,1}\right\}$.

(a) In the case of $\psi_{2, y}$, based on Table 2 ,

(2-1) $\psi_{2,1}=-1+\lambda+\mu . \quad Y_{\psi_{2,1}}=-1+Y_{\lambda}+Y_{\mu}<-1$.

$(2-2) \psi_{2,2}=\left[2 \omega_{2}\right]-1+2 \lambda+\mu . \quad\left[2 \omega_{2}\right]=0 \Rightarrow \psi_{2,2}=-1+2 \lambda+\mu . \quad Y_{\psi_{2,2}}=$ $-1+2 Y_{\lambda}+Y_{\mu}<-1 .\left[2 \omega_{2}\right]=1 \Rightarrow \psi_{2,2}=2 \lambda+\mu>\psi_{8,1}$.

$(2-3)\left[d \omega_{2}\right] \geq 1 \Rightarrow \psi_{2, d}=\left[d \omega_{2}\right]-1+d \lambda+\mu>\psi_{8,1} . \quad\left[d \omega_{2}\right]=0 \Rightarrow \psi_{2, d}=$ $-1+d \lambda+\mu . \quad Y_{\psi_{2, d}}=-1+d Y_{\lambda}+Y_{\mu}<-1$.

(2-4) $\psi_{2,2 d+1}>\psi_{8,1}$. (2-5) Similar to (2-3).

(2-6) $\psi_{2,3 d+2}>\psi_{8,1}$.

(b) In the case of $\psi_{4, y}$, based on Table 4 ,

(4-2) $\psi_{4,2}>\psi_{8,1}$. (4-3) $\psi_{4, d+1}>\psi_{8,1}$.

(4-4) $\psi_{4,2 d+1}>\psi_{8,1}$. (4-5) $\psi_{4,3 d+2}>\psi_{8,1}$.

(c) In the case of $\psi_{7, y}$, based on Table 7,

$$
\psi_{7,1}=1+\lambda-\mu<\lambda<1 .
$$

As a result, $\psi_{8,1}$ remains. 
THEOREM 6.3B. Let $\mathscr{R}=\langle 1, \lambda, \mu\rangle$ be a reduced lattice of $K$ such that $0<\lambda<1,0<X_{\mu}<X_{\lambda}, 0<\omega_{1}(\lambda, \mu)<1, \omega_{2}(\lambda, \mu)>0, a>1,2|b|<1, \mu<0$, $\phi_{1}<1, F\left(\phi_{6}\right)<1$, where $a=F(\mu), b=Y_{\mu}$. Then

(1) If $F\left(\phi_{8}\right)<1$, then the minimal point adjacent to 1 is $\phi_{8}$.

(2) If $F\left(\phi_{8}\right)>1$ :

(i) if $2 \lambda+\mu<0$, then the minimal point adjacent to 1 is $\phi_{6}$ or $\phi_{6}+\phi_{9}$;

(ii) if $2 \lambda+\mu>0$, then the minimal point adjacent to 1 is $\phi_{6}$ or $1+\phi_{9}$.

Proof. From the assumption $\phi_{1}<1$, by Lemma 5.2,(1), we have $Y_{\lambda}<$ $-1 / 2$. Since $\mu<0$ and $0<X_{\mu}$, we have $b<0$. By Corollary 5.3, we have $\omega_{2}>1 / 2$. From Table 10 and Lemma 4.5,(3), we have $\theta_{g} \in\left\{\psi_{1, y}, \psi_{3, y}, \psi_{4, y}, \psi_{5, y}\right.$, $\left.\psi_{8, y}, \psi_{9, y}\right\}$.

(a) In the case of $\psi_{1, y}$, based on Table 1,

(1-2) $\psi_{1,2}=2 \lambda+\mu . \quad Y_{\psi_{1,2}}=2 Y_{\lambda}+Y_{\mu}<-1$.

$*(1-3) d \geq 5 \Rightarrow \psi_{1, d+1} \geq\left[6 \omega_{2}\right]-1+6 \lambda+\mu \geq 2+6 \lambda+\mu>\psi_{8,1} . \quad d=1 \Rightarrow$ $\psi_{1, d+1}=2 \lambda+\mu . \quad Y_{\psi_{1, d+1}}=2 Y_{\lambda}+Y_{\mu}<-1$.

Hence, only when $2 \leq d \leq 4$, it is possible to have $\theta_{g}=\psi_{1, d+1}$.

$*(1-4) d \geq 3 \Rightarrow \psi_{1,2 d+1} \geq\left[7 \omega_{2}\right]-1+7 \lambda+2 \mu \geq 2+7 \lambda+2 \mu>\psi_{8,1}$.

Hence, only when $1 \leq d \leq 2$, it is possible to have $\theta_{g}=\psi_{1,2 d+1}$.

$*(1-5) d \geq 2 \Rightarrow \psi_{1,3 d+2} \geq\left[8 \omega_{2}\right]-1+8 \lambda+3 \mu \geq 3+8 \lambda+3 \mu>\psi_{8,1}$.

Hence, only when $d=1$, it is possible to have $\theta_{g}=\psi_{1,2 d+1}=\psi_{1,5}$.

(b) In the case of $\psi_{3, y}$, based on Table 3,

(3-1) By Lemma 4.5,(4), $\phi_{3}=\psi_{3,1} \neq \theta_{g}$.

$*(3-2) d \geq 2 \Rightarrow \psi_{3,3 d+2}>\psi_{8,1}$. Hence, only when $d=1$, it is possible to have $\theta_{g}=\psi_{3,3 d+2}=\psi_{3,5}$.

(c) In the case of $\psi_{4, y}$, based on Table 4 ,

$*(4-2) \psi_{4,2}=1+2 \lambda+\mu$.

$*(4-3) d \geq 3 \Rightarrow \psi_{4, d+1} \geq\left[4 \omega_{2}\right]+4 \lambda+\mu \geq 2+4 \lambda+\mu>\psi_{8,1}$.

Hence, only when $1 \leq d \leq 2$, it is possible to have $\theta_{g}=\psi_{4, d+1}$.

$*(4-4) d \geq 2 \Rightarrow \psi_{4,2 d+1} \geq\left[5 \omega_{2}\right]+5 \lambda+2 \mu \geq 2+5 \lambda+2 \mu>\psi_{8,1}$.

Hence, only when $d=1$, it is possible to have $\theta_{g}=\psi_{4,2 d+1}$.

$*(4-5) d \geq 2 \Rightarrow \psi_{4,3 d+2} \geq\left[8 \omega_{2}\right]+8 \lambda+3 \mu \geq 4+8 \lambda+3 \mu>\psi_{8,1}$.

Hence, only when $d=1$, it is possible to have $\theta_{g}=\psi_{4,3 d+2}$.

(d) In the case of $\psi_{5, y}$, based on Table 5,

$*(5-2) \psi_{5,2}=1+2 \lambda+\mu$.

$*(5-3) d \geq 4 \Rightarrow \psi_{5, d} \geq\left[4 \omega_{2}\right]+4 \lambda+\mu \geq 2+4 \lambda+\mu>\psi_{8,1} . \quad d=1 \Rightarrow \psi_{5, d}=$ $\lambda+\mu<1$.

Hence, only when $2 \leq d \leq 3$, it is possible to have $\theta_{g}=\psi_{5, d}$. 
$*(5-4) d \geq 2 \Rightarrow \psi_{5,2 d+1} \geq\left[5 \omega_{2}\right]+5 \lambda+2 \mu \geq 2+5 \lambda+2 \mu>\psi_{8,1}$.

Hence, only when $d=1$, it is possible to have $\theta_{g}=\psi_{5,2 d+1}$.

$*(5-5) d \geq 5 \Rightarrow \psi_{5, d-1} \geq\left[4 \omega_{2}\right]+4 \lambda+\mu \geq 2+4 \lambda+\mu>\psi_{8,1} . d=2 \Rightarrow \psi_{5, d}=$ $\lambda+\mu<1$.

Hence, only when $3 \leq d \leq 4$, it is possible to have $\theta_{g}=\psi_{5, d-1}$.

$*(5-6) d \geq 2 \Rightarrow \psi_{5,3 d+2} \geq\left[8 \omega_{2}\right]+8 \lambda+3 \mu \geq 4+8 \lambda+3 \mu>\psi_{8,1}$.

Hence, only when $d=1$, it is possible to have $\theta_{g}=\psi_{5,3 d+2}$.

(e) In the case of $\psi_{8, y}$, based on Table 8 ,

*(8-1) From the assumption, $F\left(\psi_{8,1}\right)<1$.

(f) In the case of $\psi_{9, y}$, based on Table 9,

$*(9-1) \psi_{9,1}=\left[\omega_{2}\right]+1+\lambda+\mu$.

From described above, we shall select all the elements in each part with asterisk $\left(^{*}\right)$, using $1 \leq\left[3 \omega_{2}\right] \leq 2,2 \leq\left[4 \omega_{2}\right] \leq 3,2 \leq\left[5 \omega_{2}\right] \leq 4$. Then we have the following set

$$
\begin{aligned}
& \{1+\lambda, 1+\lambda+\mu, 1+2 \lambda+\mu, j+3 \lambda+\mu(0 \leq j \leq 2), \\
& \quad j+3 \lambda+2 \mu(0 \leq j \leq 2), j+4 \lambda+\mu(1 \leq j \leq 2), j+5 \lambda+\mu(1 \leq j \leq 3), \\
& \quad j+5 \lambda+2 \mu(1 \leq j \leq 3), j+5 \lambda+3 \mu(1 \leq j \leq 4)\}=\Sigma .
\end{aligned}
$$

Here, we eliminate elements $\psi \in \Sigma$ such that $\psi>\phi_{6}$ or $Y_{\psi}<-1$. Then we have

$$
\Sigma^{\prime}=\{1+\lambda, 1+\lambda+\mu, 1+2 \lambda+\mu, 1+3 \lambda+\mu, 1+3 \lambda+2 \mu, 2+5 \lambda+3 \mu\} .
$$

(1) We assume that $F\left(\phi_{8}\right)<1$. Since $\mathscr{R}$ is a reduced lattice, we have $\phi_{8}=$ $\psi_{9,1}=1+\lambda+\mu>1$. Hence, we have $\lambda+\mu>0$. From this, we have $1+\lambda+\mu$ $<1+2 \lambda+\mu, 1+3 \lambda+\mu, 1+3 \lambda+2 \mu, 2+5 \lambda+3 \mu$. Therefore we conclude that $\theta_{g}=\phi_{8}=1+\lambda+\mu$ because $\phi_{8}<\phi_{6}=1+\lambda$.

(2) We assume that $F\left(\phi_{8}\right)>1$. We note that $d(\lambda, \mu)=1 \Leftrightarrow 1 / 2<\omega_{1}$. Hence, if $d=1$, then by Lemma 4.5,(11), we have $F\left(\phi_{8}\right)<1$. Therefore we have $d \geq 2$. So we have $\theta_{g} \neq 1+3 \lambda+2 \mu, 2+5 \lambda+3 \mu$.

(i) The case $2 \lambda+\mu<0$. We have $\theta_{g}=1+\lambda$ or $1+3 \lambda+\mu$.

(ii) The case $2 \lambda+\mu>0$. We have $\theta_{g}=1+\lambda$ or $1+2 \lambda+\mu$.

\section{Examples}

\section{Voronoi-algorithm:}

Let $K$ be a cubic algebraic number field of negative discriminant and let $\mathscr{R}$ be a reduced lattice of $K$. We define the increasing chain of the minimal points 
of $\mathscr{R}$ by:

$$
\theta_{0}=1, \quad \theta_{k+1}=\min \left\{\gamma \in \mathscr{R} ; \theta_{k}<\gamma, F\left(\theta_{k}\right)>F(\gamma)\right\} \quad \text { if } k \geq 0 .
$$

Then $\theta_{k+1}$ is the minimal point adjacent to $\theta_{k}$ in $\mathscr{R}$.

Let $\mathcal{O}_{K}$ be the ring of integers in $K$ and $\mathscr{R}=\mathcal{O}_{K}$. By Voronoi we know that the previous chain is of purely periodic form:

$$
1=\theta_{0}, \theta_{1}, \ldots, \theta_{\ell-1}, \epsilon, \epsilon \theta_{1}, \ldots, \epsilon \theta_{\ell-1}, \ldots,
$$

where $\ell$ denotes the period length and $\epsilon(>1)$ is the fundamental unit of $\mathcal{O}_{K}$. To calculate such a sequence, it is sufficient to know how to find the minimal point adjacent to 1 in a lattice $\mathscr{R}$.

Indeed, let $\theta_{g}^{(1)}$ be the minimal point adjacent to 1 in $\mathscr{R}_{1}=\mathcal{O}_{K}=\langle 1, \beta, \gamma\rangle$ and $\theta_{1}=\theta_{g}^{(1)}$.

(i) We choose an appropriate point $\theta_{h}^{(1)}$ so that $\left\{1, \theta_{g}^{(1)}, \theta_{h}^{(1)}\right\}$ is a basis of $\mathscr{R}_{1}$.

(ii) Let $\mathscr{R}_{2}=\frac{1}{\theta_{g}^{(1)}} \mathscr{R}_{1}$, then $\mathscr{R}_{2}$ is a reduced lattice. $\theta_{g}^{(2)}$ is the minimal point adjacent to 1 in $\mathscr{R}_{2}=\frac{1}{\theta_{g}^{(1)}} \mathscr{R}_{1}=\left\langle 1,1 / \theta_{g}^{(1)}, \theta_{h}^{(1)} / \theta_{g}^{(1)}\right\rangle$, is equivalent to $\theta_{2}=\theta_{1} \theta_{g}^{(2)}$ $=\theta_{g}^{(1)} \theta_{g}^{(2)}$ being the minimal point adjacent to $\theta_{1}$ in $\mathscr{R}_{1}$.

This process can be continued by induction.

Example 7.1. Let $K=\mathbf{Q}(\theta)$ be a cubic number field defined by $\theta^{3}-7 \theta-12=0(\theta=3.2669)$. Then $\mathscr{R}_{8}=\left\langle 1,-2+\frac{1}{6} \theta+\frac{1}{6} \theta^{2}, 2+\frac{2}{3} \theta-\frac{1}{3} \theta^{2}\right\rangle=$
$\langle 1, \lambda, \mu\rangle$.

It is easily seen that $0<\lambda<1,0<\mu<1$.

Since $\mathscr{R}_{8}$ is a reduced lattice, we have $a=F(\mu)>1$.

$$
\begin{aligned}
& Y_{\theta}=\frac{1}{2}\left(T_{K / \mathbf{Q}} \theta-\theta\right)=-\frac{1}{2} \theta, \quad Y_{\theta^{2}}=\frac{1}{2}\left(T_{K / \mathbf{Q}} \theta^{2}-\theta^{2}\right)=\frac{1}{2}\left(14-\theta^{2}\right) . \\
& X_{\theta}=\frac{1}{2}\left(3 \theta-T_{K / \mathbf{Q}} \theta\right)=\frac{3}{2} \theta, \quad X_{\theta^{2}}=\frac{1}{2}\left(3 \theta^{2}-T_{K / \mathbf{Q}} \theta^{2}\right)=\frac{1}{2}\left(3 \theta^{2}-14\right) . \\
& X_{\mu}=X_{2+(2 / 3) \theta-(1 / 3) \theta^{2}}=\frac{2}{3} X_{\theta}-\frac{1}{3} X_{\theta^{2}}=\frac{7}{3}+\theta-\frac{1}{2} \theta^{2}>0, \\
& X_{\lambda}-X_{\mu}=-\frac{7}{2}-\frac{3}{4} \theta+\frac{3}{4} \theta^{2}>0 . \\
& Y_{\mu}=Y_{2+(2 / 3) \theta-(1 / 3) \theta^{2}}=2+\frac{2}{3} Y_{\theta}-\frac{1}{3} Y_{\theta^{2}}=\frac{1}{6}\left(-2-2 \theta+\theta^{2}\right), \quad 0<Y_{\mu}<\frac{1}{2} .
\end{aligned}
$$




$$
\begin{aligned}
& Y_{\lambda}=\frac{1}{12}\left(-10-\theta-\theta^{2}\right) . \quad \omega_{1}(\lambda, \mu)=\frac{\theta-1}{2(\theta+2)}, \quad 0<\omega_{1}<1 . \\
& \begin{array}{c}
\omega_{2}(\lambda, \mu)=-\frac{1}{12}\left(-10-\theta-\theta^{2}\right)-\frac{\theta-1}{2(\theta+2)} \times \frac{1}{6}\left(-2-2 \theta+\theta^{2}\right) \\
=\frac{1}{4}\left(\theta^{2}-3\right), \quad\left[\omega_{2}\right]=1 . \\
F\left(\left[\omega_{2}\right]+\lambda\right)=F(1+\lambda)=1+\frac{1}{2}(\theta-3)>1 . \\
F\left(\left[\omega_{2}\right]+\lambda+\mu\right)=F(1+\lambda+\mu)=2-5 \theta+\theta^{2}+\frac{50}{\theta}>1 . \\
F\left(\left[\omega_{2}\right]+1+\lambda\right)=F(2+\lambda)=F\left(\frac{1}{6} \theta+\frac{1}{6} \theta^{2}\right)=\frac{1}{3 \theta^{2}}\left(12+\theta-\theta^{2}\right)<1 .
\end{array}
\end{aligned}
$$

Therefore, by Theorem $6.1 \mathrm{~A},(3)$, we have $\theta_{g}=\left[\omega_{2}\right]+1+\lambda=2+\lambda$.

ExAmple 7.2. Let $K=\mathbf{Q}(\theta)$ be a cubic number field defined by $\theta^{3}-2 \theta-111=0(\theta=4.9445)$. Then

$$
\mathscr{R}_{7}=\left\langle 1,\left(-71+15 \theta+\theta^{2}\right) / 98,\left(-61-23 \theta+5 \theta^{2}\right) / 196\right\rangle=\langle 1, \lambda, \mu\rangle .
$$

It is easily seen that $0<\lambda<1, \mu<0$.

Since $\mathscr{R}_{7}$ is a reduced lattice, we have $a=F(\mu)>1$.

$$
\begin{aligned}
& X_{\theta}=\frac{3}{2} \theta, \quad X_{\theta^{2}}=\frac{1}{2}\left(3 \theta^{2}-4\right) . \\
& X_{\mu}=\frac{1}{2 c}\left(15 \theta^{2}-69 \theta-20\right)=0.0141>0 \quad(c=196) . \\
& X_{\lambda}-X_{\mu}=\frac{1}{2 c}\left(-9 \theta^{2}+159 \theta+12\right)=1.4748>0 . \\
& Y_{\mu}=\frac{1}{2 c}\left(-5 \theta^{2}+23 \theta-102\right)=-0.2819, \quad 0<\left|Y_{\mu}\right|<\frac{1}{2} . \\
& Y_{\lambda}=\frac{1}{2 \times 98}\left(-\theta^{2}-15 \theta-138\right)=\frac{1}{c}\left(-\theta^{2}-15 \theta-138\right)=-1.2072 . \\
& \omega_{1}(\lambda, \mu)=\frac{-2 \theta+30}{5 \theta+23}=0.4214, \quad 0<\omega_{1}<1 . \\
& \omega_{2}(\lambda, \mu)=-Y_{\lambda}-\omega_{1} Y_{\mu}=1.2072-0.4214 \times-0.2819, \quad\left[\omega_{2}\right]=1 .
\end{aligned}
$$


(1) $N_{K / \mathbf{Q}}\left(x+y \theta+z \theta^{2}\right)=x^{3}+2 \times 2 x^{2} z-2 x y^{2}-3 \times 111 x y z+2^{2} x z^{2}+111 y^{3}$ $-2 \times 111 y z^{2}+111^{2} z^{3}$.

(a) By (1),

$$
\begin{aligned}
F\left(\phi_{1}\right) & =F\left(\left[\omega_{2}\right]+\lambda\right)=F\left(\frac{1}{98}\left(27+15 \theta+\theta^{2}\right)\right) \\
& =\frac{1}{98^{2}} F\left(27+15 \theta+\theta^{2}\right)=\frac{1}{98^{2}} \frac{N_{K / \mathbf{Q}}\left(27+15 \theta+\theta^{2}\right)}{27+15 \theta+\theta^{2}} \\
& =\frac{1}{98^{2}} \frac{259308}{27+15 \theta+\theta^{2}}=0.2149<1 .
\end{aligned}
$$

(b) $\lambda+\mu=\frac{1}{c}\left(7 \theta^{2}+7 \theta-203\right)=\frac{1}{c} \times 2.7480>0$.

(c) By (1),

$$
\begin{aligned}
F\left(\phi_{2}\right) & =F\left(\left[\omega_{2}\right]+\lambda+\mu\right)=F\left(\frac{1}{c}\left(-7+7 \theta+7 \theta^{2}\right)\right) \\
& =\frac{1}{c^{2}} F\left(-7+7 \theta+7 \theta^{2}\right)=\frac{1}{c^{2}} \frac{N_{K / \mathbf{Q}}\left(-7+7 \theta+7 \theta^{2}\right)}{-7+7 \theta+7 \theta^{2}} \\
& =\frac{1}{c^{2}} \frac{4302592}{-7+7 \theta+7 \theta^{2}}=0.5635<1 .
\end{aligned}
$$

Therefore, by Theorem 6.3A,(1),(ii-b), we have $\theta_{g}=\phi_{2}$.

ExAmple 7.3. Let $K=\mathbf{Q}(\theta)$ be a cubic number field defined by $\theta^{3}-77 \theta-$ $513=0(\theta=11.1002)$. Then

$$
\mathscr{R}_{39}=\left\langle 1,\left(-674-28 \theta+9 \theta^{2}\right) / 613,\left(1205+121 \theta-17 \theta^{2}\right) / 613\right\rangle=\langle 1, \lambda, \mu\rangle .
$$

It is easily seen that $0<\lambda<1,0<\mu<1$.

Since $\mathscr{R}_{39}$ is a reduced lattice, we have $a=F(\mu)>1$.

$$
\begin{aligned}
& X_{\theta}=\frac{3}{2} \theta, \quad X_{\theta^{2}}=\frac{1}{2}\left(3 \theta^{2}-154\right) . \\
& X_{\mu}=\frac{1}{2 c}\left(-51 \theta^{2}+363 \theta+2618\right)=\frac{1}{2 c} \times 363.4361>0 \quad(c=613) . \\
& X_{\lambda}-X_{\mu}=\frac{1}{2 c}\left(78 \theta^{2}-457 \theta-4004\right)=\frac{1}{2 c} \times 533.9349>0 . \\
& Y_{\mu}=\frac{1}{2 c}\left(17 \theta^{2}-121 \theta-208\right)=0.4433, \quad 0<Y_{\mu}<\frac{1}{2} .
\end{aligned}
$$




$$
\begin{aligned}
& Y_{\lambda}=\frac{1}{2 c}\left(-9 \theta^{2}+28 \theta+38\right)=-0.6200 . \quad \omega_{1}(\lambda, \mu)=\frac{9 \theta+28}{17 \theta+121}=0.4129 \\
& 0<\omega_{1}<1 . \quad \omega_{2}(\lambda, \mu)=-Y_{\lambda}-\omega_{1} Y_{\mu}=0.6200-0.4129 \times 0.4433, \quad\left[\omega_{2}\right]=0 .
\end{aligned}
$$

(a) $\phi_{2}=\lambda+\mu=\frac{1}{c}\left(-8 \theta^{2}+93 \theta+521\right)=0.9259<1$.

(b) $2 \lambda+\mu=\frac{1}{c}\left(\theta^{2}+65 \theta-143\right)=1.1447>1$.

(1) $N_{K / \mathbf{Q}}\left(x+y \theta+z \theta^{2}\right)=x^{3}+2 \times 77 x^{2} z-77 x y^{2}-3 \times 513 x y z+77^{2} x z^{2}+$ $513 y^{3}-77 \times 513 y z^{2}+513^{2} z^{3}$.

(c) By (1),

$$
\begin{aligned}
F\left(\phi_{6}\right) & =F\left(\left[\omega_{2}\right]+1+\lambda\right)=F\left(\frac{1}{c}\left(-61-28 \theta+9 \theta^{2}\right)\right) \\
& =\frac{1}{c^{2}} F\left(-61-28 \theta+9 \theta^{2}\right)=\frac{1}{c^{2}} \frac{N_{K / \mathbf{Q}}\left(-61-28 \theta+9 \theta^{2}\right)}{-61-28 \theta+9 \theta^{2}} \\
& =\frac{1}{c^{2}} \frac{225837169}{-61-28 \theta+9 \theta^{2}}=0.8153<1 .
\end{aligned}
$$

(d) By (1),

$$
\begin{aligned}
F(2 \lambda+\mu) & =\frac{1}{c^{2}} F\left(\theta^{2}+65 \theta-143\right)=\frac{1}{c^{2}} \frac{N_{K / \mathbf{Q}}\left(\theta^{2}+65 \theta-143\right)}{\theta^{2}+65 \theta-143} \\
& =\frac{1}{c^{2}} \frac{198781801}{\theta^{2}+65 \theta-143}=0.7538<1 .
\end{aligned}
$$

Therefore, by Theorem 6.1B,(3),(ii-b), we have $\theta_{g}=2 \lambda+\mu$.

EXAMPLe 7.4 (Williams and Dueck [8, p. 690]). Let $K=\mathbf{Q}(\theta)$ be a cubic number field defined by $\theta^{3}-68781=0(\theta=40.97221992)$. Then

$$
\begin{aligned}
& \mathscr{R}_{2307}=\langle 1, \phi, \psi\rangle \\
&=\langle 1,(-72036+\left.\left.1809 \theta+2 \theta^{2}\right) / 126539,\left(117574-2668 \theta+67 \theta^{2}\right) / 126539\right\rangle \\
&=\langle 1, \phi, \psi-1\rangle=\left\langle 1,\left(-72036+1809 \theta+2 \theta^{2}\right) / 126539,\right. \\
&\left.\quad\left(-8965-2668 \theta+67 \theta^{2}\right) / 126539\right\rangle \\
&=\langle 1, \lambda, \mu\rangle . \quad 0<\lambda<1, \mu<0 . \quad 0<X_{\mu}<X_{\lambda} .
\end{aligned}
$$


Since $\mathscr{R}_{2307}$ is a reduced lattice, we have $a=F(\mu)>1$.

$$
\begin{aligned}
& \omega_{1}(\lambda, \mu)=\frac{-2 \theta+1809}{67 \theta+2668} . \quad Y_{\lambda}=-\frac{1}{2 c}\left(2 \theta^{2}+1809 \theta+144072\right) \quad(c=126539) . \\
& Y_{\mu}=-\frac{1}{2 c}\left(67 \theta^{2}-2668 \theta+17930\right) . \\
& \omega_{1}=0.31904891 . \quad Y_{\lambda}=-0.87541450 . \quad Y_{\mu}=-0.08333592 . \\
& \omega_{2}=0.90200274 .
\end{aligned}
$$

Hence $\left[\omega_{2}\right]=0, \phi_{1}=\left[\omega_{2}\right]+\lambda=\lambda<1$.

(1) $N_{K / \mathbf{Q}}\left(x+y \theta+z \theta^{2}\right)=x^{3}-3 \times 68781 x y z+68781 y^{3}+68781^{2} z^{3}$.

(a) By (1),

$$
\begin{aligned}
F\left(\phi_{6}\right) & =F\left(\left[\omega_{2}\right]+1+\lambda\right)=F(1+\lambda)=F\left(\frac{1}{c}\left(54503+1809 \theta+2 \theta^{2}\right)\right) \\
& =\frac{1}{c^{2}} F\left(54503+1809 \theta+2 \theta^{2}\right)=\frac{1}{c^{2}} \frac{N_{K / \mathbf{Q}}\left(54503+1809 \theta+2 \theta^{2}\right)}{54503+1809 \theta+2 \theta^{2}} \\
& =\frac{1}{c^{2}} \frac{528431935430042}{54503+1809 \theta+2 \theta^{2}}=0.25005464<1 .
\end{aligned}
$$

(b) By (1),

$$
\begin{aligned}
F(1+2 \lambda+\mu) & =F\left(\frac{-26498+950 \theta+71 \theta^{2}}{c}\right) \\
& =\frac{1}{c^{2}} F\left(-26498+950 \theta+71 \theta^{2}\right)=\frac{1}{c^{2}} \frac{N_{K / \mathbf{Q}}\left(-26498+950 \theta+71 \theta^{2}\right)}{-26498+950 \theta+71 \theta^{2}} \\
& =\frac{1}{c^{2}} \frac{2102375149688779}{-26498+950 \theta+71 \theta^{2}}=0.99760062<1 .
\end{aligned}
$$

(c) By (1),

$$
\begin{aligned}
F\left(\phi_{8}\right) & =F(1+\lambda+\mu)=F\left(\frac{45538-859 \theta+69 \theta^{2}}{c}\right) \\
& =\frac{1}{c^{2}} F\left(45538-859 \theta+69 \theta^{2}\right)=\frac{1}{c^{2}} \frac{N_{K / \mathbf{Q}}\left(45538-859 \theta+69 \theta^{2}\right)}{45538-859 \theta+69 \theta^{2}} \\
& =\frac{1}{c^{2}} \frac{2161892194231336}{45538-859 \theta+69 \theta^{2}}=1.07007239>1 .
\end{aligned}
$$


(d) Since $-153037+950 \theta+71 \theta^{2}>0,2 \lambda+\mu=\frac{-153037+950 \theta+71 \theta^{2}}{c}>0$.

(e) Since $\lambda+\mu=\frac{-81001-859 \theta+69 \theta^{2}}{c}<0$, we have $1+2 \lambda+\mu<1+\lambda$.

Therefore, by Theorem $6.3 \mathrm{~B},(2)$,(ii), we have $\theta_{g}=1+2 \lambda+\mu$.

\section{Acknowledgment}

I would like to thank the referee for his/her careful reading of the original manuscript and many helpful suggestions.

\section{References}

[1] B. Adam, Voronoi-algorithm expansion of two families with period length going to infinity, Math. Comp. 64 (1995), 1687-1704.

[2] B. N. Delone and D. K. Faddeev, The theory of irrationalities of the third degree, Transl. Math. Monographs, vol. 10, Amer. Math. Soc., Providence, RI, 1964.

[ 3 ] K. Kaneko, On the cubic fields $\mathbf{Q}(\theta)$ defined by $\theta^{3}-3 \theta+b^{3}=0$, Sut J. Math. vol. 32, No. 2 (1996), 141-147.

[4] K. Kaneko, Voronoi-algorithm expansion of a family with period length going to infinity, Sut J. Math. vol. 34, No. 1 (1998), 49-62.

[ 5 ] O. Lahlou and A. Farhane, Sur les points extrémaux dans un ordre cubique, Bull. Belg. Math. Soc. 12 (2005), 449-459.

[6] H. C. Williams, G. Cormack and E. Seah, Calculation of the regulator of a pure cubic field, Math. Comp. 34 (1980), 567-611.

[ 7 ] H. C. Williams, G. W. Dueck and B. K. Schmid, A rapid method of evaluating the regulator and class number of a pure cubic field, Math. Comp. 41 (1983), 235-286.

[ 8 ] H. C. Williams and G. W. Dueck, An analogue of the nearest integer continued fraction for certain cubic irrationalities, Math. Comp. 42 (1984), 683-705.

[ 9 ] H. C. Williams, Continued fractions and number-theoretic computations, Rocky Mountain J. Math. 15 (1985), 621-655.

[10] H. C. Williams, The period length of Voronoi's algorithm for certain cubic orders, Publ, Math. Debrecen 37 (1990), 245-265.

Graduate School of Mathematics

University of Tsukuba

1-1-1 Tennohdai, Tsukuba, Ibaraki 305-8573, Japan 